\title{
Taylors' Technique for Solving Delay Equations of Gene Expression Model with Competitive and Non- Competitive Mechanisms
}

\author{
Angham A. Jabar and A. S. J. Al- Saif \\ Department of Mathematics, College of Education for Pure Sciences, University of Basrah,Basrah, Iraq
}

\begin{abstract}
In this paper, the delay differential equations of Gene expression models with mechanisms of signal-dependent transcription regulation are solved and studied in two cases: When there is (i) competition and (ii) without competition(non-competition) for Deoxy ribo Nucleic Acid (DNA) regulatory binding sites in a cell. Also, we studied the effect of both increasing the inhibitor or decreasing the abundance of the activator (inhibition mechanism), and decreasing the inhibitor or increasing the abundance of the activator (activator mechanism) on the steady-state of the solutions. A new analytical approximation approach derived from Taylor series expansion is used for solving the delay differential equations of gene expression models. From the analytical approximate solutions of gene expression models that are resulting from using the proposed method, we found that the behavior of the solution in the activation mechanisms whether in the competitive or non-competitive model is more stable than the abundance of the activator increases, while the inhibition mechanisms are less stable. We also noticed that the convergence of these solutions is achieved with a few iterations.
\end{abstract}

Keywords: Taylors' technique, activator, inhibitor, Gen, mRNA, protein, convergence analysis.

DOI: $10.7176 / \mathrm{MTM} / 12-1-01$

Publication date: January $31^{\text {st }} 2022$

\section{1-Introduction}

Gene expression is a process by which genetic information is used to obtain genetic products or a type of Ribonucleic Acid (RNA). Features of gene expression in cells are rarely homogeneous in time and space. Dynamic time-domain behaviors such as oscillations and pulses of protein production are pervasive in cell biology and fundamental phenomena such as circadian rhythms and cell cycle control, etc. In multicellular populations, spatial changes are essential for decision-making and development among many other functions. The temporal and spatial characterization of gene expression is useful from a modeling point of view although it is experimentally and quantitatively challenging at the individual cell level, because it poses strong limitations that can be examined through theoretical analysis of mathematical models of candidate genes and protein circuits. a mathematical model of gene expression can be defined using mathematical language in different kinds of differential equations by many researcher such as, Sarkar et al. [1] who studied delayed reactions in gene regulation, They took both analytical and digital circuit models with and without delay for changing parameters and delay lengths. Their theoretical and experimental study of the variation in the transient dynamic features with increasing delay contributed helped to determine the opposite effects of the instability caused by the delay, and the stability-enhancing property of negative feedback in the behavior of pathway. They demonstrated the rationale for the abundance of similar designs in real biochemical pathways. Wang et al. [2] introduced a genetic transcriptional regulatory model, subject to associated noise and the role of time delay in gene switching with random resonance, then took two cases of time delay: the first is the linear delay that occurs during the degradation process and the second is the non-linear delay that occurs during the synthesis process. Theoretical and numerical results showed that increase in the time delay can speed up the transition from "on" to "off". They concluded that the random resonance can be improved by the time delay and the intensity of the associated noise. Necenttin et al. [3] used differential delay equations models where they studied two sets of signal-dependent transcriptional mechanisms. In the first group, the inhibitory and activator proteins competed to bind the same regulatory site on DNA (competitive mechanisms), while in the second group, the association with different regulatory site on DNA (non-competitive mechanisms). Then they studied the dynamics of gene expression on the mechanisms of inhibition and activation mechanisms. The results showed that the response time was slower in the mechanism of competitive inhibition by increasing the abundance of the repressor, and faster in the mechanism of noncompetitive inhibition due to increased abundance of the repressor, while competitive and non-competitive activation mechanisms show a more effective and faster response by increasing the abundance of the activated protein. Sharma et al. [4] took the gene expression model of strain E. coli (TJK16) which consists of a system of differential equations independent of the delay time that occurs during the process of transcription and translation, the solution was found using the fuzzy method of gene expression and noticed that when the translation rate and transcription rate values change, the steady-state of the model solutions are satisfied.

By using distinct transcription factor binding patterns in the regulatory region of genes, a cell can achieve 
similar or different responses. For example, when there is an activator and an inhibitor protein regulating the same gene, the level of gene expression can be controlled in a competitive or non-competitive manner. Cells use both mechanisms in different genes in a specific way to maintain cellular integrity and to produce appropriate responses to external stimuli. As we mentioned previously in [4], the fuzzy analytical method was applied to a gene expression model that does not care about the time of the transcription and translation delay. The system, that we use in our study, will address this problem as it contains delay equations in addition to studying two sets of signaldependent transcriptional regulation mechanisms. First, we will assume that the inhibitor and activator proteins compete for binding to the same regulatory site on DNA (the competitive mechanisms) and then the inhibitor and activator proteins bind to different regulatory regions (non-competitive mechanisms). It was used previously [3], but now we will use it to develop the model used in [4] and apply it to E. Coli (TJK16). In both case, we study the mechanisms of inhibition(increasing the repressor or decreasing the abundance of the activator), and the mechanisms of activation (decreasing the inhibitor or increasing the abundance of the activator). Therefore, this work can be considered as an extension to the study of gene expression model in [4], and the modified gene expression model that resulting from expansion process is handled by apply a new analytical approximation method [5,6,7]. This method adopted Taylor expansion to find approximate solutions for different problems, especially those whose exact solution is difficult to find. In these references, the method has proven its efficiency and its significant ability to process various models of linear and non-linear differential equations, as well as giving solutions with high-precision results and good convergence in comparison with the results of analytic exact solutions or the experimental results that obtained by different methods[8-16].

After finding the analytical solutions, using the proposed method [5], we concluded that the activation mechanisms are more stable when the abundance of the activated protein increases in both competitive and noncompetitive mechanisms. In the case of competitive and non-competitive inhibition mechanisms, there is a fluctuation in the solutions, but in competitive mechanism. It is better in the case of increasing the inhibitory protein, while in non-competitive mechanisms, reducing the activated protein is better.

After finding the analytical solutions using the proposed method [5], we concluded that the activation mechanisms in both case (competitive and non-competitive) are more stable than the inhibition mechanisms, especially we note this stability in the case of increased abundance of the activated protein, as there is fluctuation in solutions in the competitive and non-competitive inhibition mechanisms, in competitive mechanisms, solutions are more stable if the abundance of the inhibitory protein is increased, while in non-competitive mechanisms, the stability of solutions is better in the case of reducing the abundance of the activated protein.

\section{2-Mathematicalmodel:}

In our research, we will study a model of gene expression in two states, the first, the activating and repressive proteins competing to bind to the same regulatory sites of the DNA. In the second case, the activating and repressor proteins do not compete for binding, as each binds to different regulatory regions of the DNA.

\section{1-Gene regulation with competition.}

The equations below represent the gene expression model for differential delay equations in the first case when there is competition for regulatory binding sites.

$$
\begin{aligned}
& \frac{d G^{A}}{d t}=a_{0} G^{\prime}(t) A(t)-a_{f} G^{A}(t) \\
& \frac{d G^{R}}{d t}=r_{0} G^{\prime}(t) R(t)-r_{f} G^{R}(t) \\
& \frac{d M}{d t}=\eta_{m a} G_{\tau m}^{A}+\eta_{m 0} G_{\tau m}^{\prime}-\beta_{m} M \\
& \frac{d p}{d t}=\alpha_{p} M_{\tau p}-\beta_{p} P
\end{aligned}
$$

where $G^{A}, G^{R}, M$ and $P$ represent the levels of repressor binding gene, activator binding gene, mRNA abundance and protein abundance respectively. The terms $\left(a_{0}, r_{0}\right)$ and $\left(a_{f}, r_{f}\right)$ represent the binding and unbinding rate constants for the activating molecule $(A)$ and the repressor molecule $(R)$ of the gen $\left(G^{\prime}\right)$ 
respectively. $\left(\eta_{m a}, \eta_{m 0}\right.$ and $\left.\alpha_{p}\right)$ synthesis rate constants of mRNA and protein synthesis, $\left(\beta_{m}, \beta_{p}\right)$ degradation rate constants of mRNA and protein. In equation $(2.1)$ the terms $\left(a_{0} G^{\prime}(t) A(t)\right)$ and $\left(a_{f} G^{A}(t)\right)$ represent rates of building and unbinding of the activator protein. In equation (2.2) the terms $\left(r_{0} G^{\prime}(t) R(t)\right)$ represents the binding rate and $\left(r_{f} G^{R}(t)\right.$ ) the unbinding rate of the repressor protein. We assume that the total gene is

$$
G^{\prime}+G^{A}+G^{R}=1
$$

Equation (2.3) the change in mRNA concentration is the difference between its synthesis rates $\left(\eta_{m a} G_{\tau m}^{A}+\eta_{m 0} G_{\tau m}^{\prime}\right)$ and degradation rates $\left(\beta_{m} M\right)$. When looking at the mRNA synthesis rate, we find that the mRNA synthesis rate in the free $\left(\eta_{m 0} G_{\tau m}^{\prime}\right)$ and activated bound $\left(\eta_{m a} G_{\tau m}^{A}\right)$. While equation (2.4) mean that the change in protein concentration is the difference of its synthesis rates $\left(\alpha_{p} M_{\tau p}\right)$ and degradation rates $\left(\beta_{p} P\right)$.

\section{2-Gene regulation without competition}

After we have clarified the first case (the activating and inhibitory protein compete to bind the same regulatory sites of the DNA). Now for the second case, the activating and inhibitory protein do not compete to bind to the same regulatory regions of the DNA, where bind to different regulatory regions and this can be seen when looking at the following model and comparing with the previous model (2.1-2.4), which contains different boundaries for the binding sites

Now the activating and repressor proteins bind to different regulatory regions of the DNA.

$$
\begin{aligned}
& \frac{d G^{A}}{d t}=a_{0} G^{\prime}(t) A(t)+a r_{f} G^{A R}-a r_{0} R(t) G^{A}(t)-a_{f} G^{A} \\
& \frac{d G^{R}}{d t}=r_{0} G^{\prime}(t) R(t)+r a_{f} G^{A R}-r a_{0} A(t) G^{R}(t)-r_{f} G^{R} \\
& \frac{d M}{d t}=\alpha_{m a r} G_{\tau m}^{A R}+\alpha_{m a} G_{\tau m}^{A}+\alpha_{m 0} G_{\tau m}^{\prime}-\beta_{m} M \\
& \frac{d p}{d t}=\alpha_{p} M_{\tau p}-\beta_{p} P
\end{aligned}
$$

where $G^{A}, G^{R}, M$ and $P$ represent the levels of activator binding gene, repressor binding gene, mRNA abundance and protein abundance respectively. The terms $\left(a r_{0}, a r_{f}\right)$ represent the building and unbinding rates constant the activator molecule (A) of the gene $\left(G^{A}\right)$, while $\left(r a_{0}, r a_{f}\right)$ represent the building and unbinding rates constant the repressor molecule $(R)$ of the gene $\left(G^{R}\right)$. The terms $\left(\alpha_{m a}, \alpha_{m 0}\right.$ and $\left.\alpha_{p}\right)$ represent synthesis rate constants of $\left(G^{\prime}, G^{A}, G^{A R}\right)$ and protein respectively. The terms $\left(\beta_{m}, \beta_{p}\right)$ degradation rate constants of mRNA and protein.

In equation (2.6) contains the gain rate $\left(a_{0} G^{\prime}(t) A(t)+a r_{f} G^{A R}\right)$ is the sum the binding of activated protein (A) to free protein $\left(G^{\prime}\right)$ and the unbinding of activated protein $(R)$ from $\left(G^{A R}\right)$. And the loss rate $\left(-a r_{0} R(t) G^{A}(t)-a_{f} G^{A}\right)$ is the rate of the binding repressor protein (R) with the $\left(G^{A}\right)$ and the rate of the dissociation activated protein $(A)$ from the $\left(G^{A}\right)$. 
Equations (2.6) and (2.7) gain rate $\left(r_{0} G^{\prime}(t) R(t)+r a_{f} G^{A R}\right)$ is the sum the binding of repressor protein (R) to gen $\left(G^{\prime}\right)$ and the unbinding of activated protein $(A)$ from $\left(G^{A R}\right)$. The rate of loss $\left(-r a_{0} A(t) G^{R}(t)-r_{f} G^{R}\right)$ is the binding activated protein rate (A) with the $\left(G^{R}\right)$ and the rate of dissociation of the repressor protein $(\mathrm{R})$ from the $\left(G^{R}\right)$.

We assume that the total gene is

$$
G^{\prime}+G^{A}+G^{R}+G^{A R}=1
$$

In equation (2.8) the first term $\left(\alpha_{\text {mar }} G_{\tau m}^{A R}\right)$ of the synthesis mRNA rate $\left(\alpha_{m a r} G_{\tau m}^{A R}+\alpha_{m a} G_{\tau m}^{A}+\alpha_{m 0} G_{\tau m}^{\prime}\right)$ is represents the contribution of the $\left(G^{A R}\right)$ complex in the synthesis rate. The second term $\left(\alpha_{m a} G_{\tau m}^{A}\right)$ describes the contribution of the $\left(G^{A}\right)$ complex, while the term $\left(\alpha_{m 0} G^{\prime}{ }_{\tau m}(t)\right)$ accounts for the synthesis rate due to the free gene $G^{\prime}$ and $\left(\beta_{m} M\right)$ degradation rates. In equation (2.9), the rate of production of the protein (P) is the difference of its synthesis rate $\left(\alpha_{p} M_{\tau p}\right)$ and degradation rate $\left(\beta_{p} P\right)$ with the associated rate constants $\alpha_{p}$ and $\beta_{p}$, respectively.

The transcription and translation processes are not instantaneous processes. RNA must cross the gene and the ribosome's must extend through the mRNA to translate into the protein. Equation $G^{\prime} \tau_{m}=G^{\prime}\left(t-\tau_{m}\right)$ calculates the time taken for the entire process as $\tau_{m}$ and $\tau_{p}$ represent the terms of delay. Here parameter $\tau_{p}$ is the transcription time delay such that $M_{\tau m}=M\left(t-\tau_{m}\right)$ and the translational time delay such that $P_{\tau p}=p\left(t-\tau_{p}\right)$ The initial condition for the mathematical model (2.1-2.9) are

$G^{A}(t)=G^{A}{ }_{0}, t=0$

$G^{R}(t)=G_{0}^{R}, t=0$

$M(t)=m_{0}, t=0$

$P(t)=p_{0}, t=0$

To find the signal-dependent responses to these regulatory mechanisms, we hypothesize that levels are regulated activated protein $(A)$ and repressor protein $(R)$ by transient signal $\mathrm{S}(\mathrm{t})$

$s(t)=\left\{\begin{array}{lc}\gamma, \text { if } & 0 \leq t<k \\ 0, \text { if } & t \geq k\end{array}\right.$

$\gamma$ is the signal amplitude parameter that measures the sensitivity of the system to disturbance caused by the signal, and the signal continuity parameter $\mathrm{k}$ determines how long the signal is applied to the system. and the signal persistence parameter $\mathrm{k}$ determines how long the signal is applied to the system. To model the signal-induced suppression, we note in the competitive model, the activation level $A_{0}$ can be reduced by the signal $A(t)=$ $\frac{A_{0}}{1+s(t)}$ or the inhibitory $R_{0}$ can be increased by the signal $R(t)=R_{0}(1+s(t))$ Similarly, to model the signalinduced activation in the competitive model the level of the activator $A_{0}$ can be increased by the signal $A(t)=$ $A_{0}(1+s(t))$ or the level of the inhibitory $R_{0}$ can be increased. It is reduced by $R(t)=\frac{R_{0}}{1+s(t)}$.

\section{3-Taylors' technique and application:}

The series method can develop analytical methods in finding an exact and /or approximate analytical solution to many linear and non-linear differential equations[ $14,15,16]$. One of the series methods is Taylors' analytical technique that is introduced by $[8,17,18]$. To illustrate the basic ideas of this technique, let us consider the first order ordinary differential equation of the function $u(t)$ in the form of an operator as follows

$$
\frac{d u}{d t}=F[u]+g
$$

with the initial condition $u\left(t_{0}\right)$, where $u$ unknown function, $F[u]$ is linear and/or non-linear operator and 
$g$ is the known function. The Taylor series about $t_{0}$ for the solution $u(t)$ is defined as the following

$u(t)=a_{0}+a_{1} \Delta t+a_{2} \frac{(\Delta t)^{2}}{2 !}+a_{3} \frac{(\Delta t)^{3}}{3 !}+a_{4} \frac{(\Delta t)^{4}}{4 !}+\cdots$

Where $a_{0}=u\left(t_{0}\right), a_{1}=g+[F[u]]_{t_{0}}, a_{2}=\left[F^{\prime}[u]\right]_{t_{0}}, a_{3}=\left[F^{\prime \prime}[u]\right]_{t_{0}}, \ldots, a_{n}=\left[F^{(n-1)}[u]\right]_{t_{0}}$.

And the derivatives of $F[u]$ is defined as the following

$$
\begin{aligned}
& F[u]^{\prime}=\frac{d}{d t} F[u], \\
& F[u]^{\prime \prime}=\frac{d^{2}}{d t^{2}} F[u], \\
& F[u]^{\prime \prime \prime}=\frac{d^{3}}{d t^{3}} F[u], \\
& \vdots \\
& F[u]^{n-1}=\frac{d^{n-1}}{d t^{n-1}} F[u]
\end{aligned}
$$

where $n$ is the highest derivative of $u$.

Application of the gene model with competition.

Now, apply the above steps to find analytical approximate solution of equations activator bound gene, repressor bound gene, mRNA, Protein (2.1-2.9). The following equation we found from the relationship (2.5), which represents the rate of change in the free gene with the initial condition $G^{\prime}(0)=G^{\prime}(0)$ because we need it to find solutions to the equations(2.1-2.4):-

$$
\begin{aligned}
& \frac{d G^{\prime}}{d t}=r_{f}-\left(r_{0} R(t)+a_{0} A+r_{f}\right) G^{\prime}, \text { we have } \\
& F[t]=-\left(r_{0} R(t)+a_{0} A+r_{f}\right) G^{\prime} \\
& g=r_{f} \\
& G^{\prime}(0)=G_{0}^{\prime} \\
& G_{1}^{\prime}=\left.F\left[G^{\prime}\right]\right|_{t=0}+g=r_{f}-\left(r_{0} R(t)+a_{0} A(t)+r_{f}\right) G_{0}^{\prime} \\
& G_{2}^{\prime}=F\left[\left.G^{\prime}\right|_{t=0}=-\left(r_{0} R(t)+a_{0} A(t)+r_{f}\right) G_{1}^{\prime}\right.
\end{aligned}
$$

From the equation (3.2) we get

$$
\begin{aligned}
& G^{\prime}(t)=G_{0}^{\prime}+G_{1}^{\prime} t+G_{2}^{\prime} \frac{t^{2}}{2 !}+\ldots \\
& =G_{0}^{\prime}+\left(r_{f}-\left(r_{0} R(t)+a_{0} A(t)+r_{f}\right) G_{0}^{\prime}\right) t-\left((r 0 R(t)+a 0 A(t)+r f) G_{1}^{\prime}\right) \frac{t^{2}}{2 !}+\ldots \\
& =G_{0}^{\prime} e^{-\left(r_{0} R(t)+a_{0} A(t)+r_{f}\right) t}-\frac{r_{f}}{r_{0} R(t)+a_{0} A(t)+r_{f}}\left(e^{-\left(r_{0} R(t)+a_{0} A(t)+r_{f}\right) t}-1\right)
\end{aligned}
$$

To find the activator binding concentration, take equation (2.1) with the initial condition (2.11)

$$
\frac{d G^{A w}}{d t}=a_{0} G^{\prime}(t) A(t)-a_{f} G^{A}(t)
$$

We have

$$
\begin{aligned}
& F[t]=a_{0} G^{\prime}(t) A(t)-a_{f} G^{A}(t) \\
& g=0
\end{aligned}
$$




$$
\begin{aligned}
& G_{0}^{A w}=G^{A}(0) \\
& \quad G_{1}^{A w}=\left.F[t]\right|_{t=t_{0}}=a_{0} A(t) G_{1}^{\prime}-a_{f} G_{0}^{A} \\
& \dot{G}_{2}^{A w}=\left.F^{\prime}[t]\right|_{t=0}=a_{0} A(t) G_{2}^{\prime}-a_{f} G_{1}^{A} \text { From the equation (3.2) we get } \\
& G^{A w}(t)=G_{0}^{A w}+G_{1}^{A w} t+G_{2}^{A w} \frac{t^{2}}{2 !}+\ldots \\
& =G_{0}^{A w}+\left[a_{0} A(t)\left(r f-\left(r 0 R(t)+a_{0} A(t)+r f\right) G_{0}^{\prime}-a_{f} G_{0}^{A w}\right] t+\left[a _ { 0 } A ( t ) \left(-r f\left(r_{0} R(t)+a_{0} A(t)+r_{f}\right) G_{0}^{\prime}+\right.\right.\right. \\
& \left.a_{0} A(t) r_{f}-a_{f}\left(a_{0} A(t) G_{0}^{\prime}-a_{f} G_{0}^{A w}\right)\right] \frac{t^{2}}{2 !}+\cdots
\end{aligned}
$$

Now, we will take equation (2.2) with initial condition(2.12)

$$
\frac{d G^{R w}}{d t}=r_{0} G^{\prime}(t) R(t)-r_{f} G^{R w}(t)
$$

We have

$$
\begin{aligned}
& F[t]=r_{0} G^{\prime}(t) R(t)-r_{f} G^{R w}(t) \\
& g=0 \\
& G_{0}^{R w}=G^{R w}(0) \\
& G_{1}^{R w}=\left.F[t]\right|_{t=t_{0}}=r_{0} R(t) G_{1}^{\prime}-r_{f} G_{0}^{R w} \\
& G_{2}^{R w}=\left.F^{\prime}[t]\right|_{t=0}=r_{0} R(t) G_{2}^{\prime}-r_{f} G_{1}^{R w}
\end{aligned}
$$$$
G^{R w}(t)=G_{0}^{R w}+G_{1}^{R w} t+G_{2}^{R w} \frac{t^{2}}{2 !}+\ldots
$$$$
=G_{0}^{R w}+\left(r_{0} R(t) G_{1}^{\prime}-r_{f} G_{0}^{R w}\right) t+\left(r_{0} R(t) G_{2}^{\prime}-r_{f} G_{1}^{R w}\right) \frac{t^{2}}{2 !}+\ldots
$$

Now, we will take the equation for the change in the concentration mRNA (2.3) with the initial condition (2.13)

$$
\frac{d M^{w}}{d t}=\eta_{m a} G_{\tau m}^{A w}+\eta_{m 0} G_{\tau m}^{\prime}-\beta_{m} M^{w}
$$

We have

$$
\begin{aligned}
& F[t]=\eta_{m a} G_{\tau m}^{A w}+\eta_{m 0} G_{\tau m}^{\prime}-\beta_{m} M^{w} \\
& g=0 \\
& M_{0}^{w}=M(0) \\
& M_{1}^{w}=\left.F[t]\right|_{t=0}+g=n_{m a} G_{1}^{A w}\left(t-\tau_{m}\right)+n_{m 0} G_{1}^{\prime}\left(t-\tau_{m}\right)-B_{m} M_{0}^{w} \\
& M_{2}^{w}=\left.F^{\prime}[t]\right|_{t=0}=n_{m a} G_{2}^{A w}\left(t-\tau_{m}\right)+n_{m 0} G_{2}^{\prime}\left(t-\tau_{m}\right)-B_{m} M_{1}^{w} \vdots
\end{aligned}
$$

From the equation (3.2) we get

$$
M^{w}(t)=M_{0}^{w}+M_{1}^{w} t+M_{2}^{w} \frac{t^{2}}{2 !}+\ldots
$$




$$
\begin{gathered}
=M_{0}^{w}+\left[n_{m a} G_{1}^{A w}\left(t-\tau_{m}\right)+n_{m 0} G_{2}^{\prime}\left(t-\tau_{m}\right)-B_{m} M_{0}^{w}\right] t+\left[n_{m a} G_{2}^{A w}\left(t-\tau_{m}\right)+\right. \\
\left.n_{m 0} G_{2}^{\prime}\left(t-\tau_{m}\right)-B_{m} M_{1}^{w}\right] \frac{t^{2}}{2 !}+\ldots
\end{gathered}
$$

Now, we will take the equation for the change in the concentration protein (2.4) with the initial condition (2.14)

$\frac{d p^{w}}{d t}=\alpha_{p} M_{\tau p}{ }^{w}-\beta_{p} P^{w}$ we have

$F[t]=\alpha_{p} M_{\tau p}{ }^{w}-\beta_{p} P^{w}$

$g=0$

$p_{0}^{w}=p(0)$

$p_{1}^{w}=\left.F[t]\right|_{t=0}=\alpha_{p} m_{1}^{w}\left(t-\tau_{p}\right)-\beta_{p} p_{0}^{w}$

$p_{2}^{w}=\left.F^{\prime}[t]\right|_{t=0}=\alpha_{p} m_{2}^{w}\left(t-\tau_{p}\right)-\beta_{p} p_{1}^{w}$

$\vdots$

From the equation (3.2) we get

$$
\begin{aligned}
& p^{w}(t)=p_{0}^{w}+p_{1}^{w} t+p_{2}^{w} \frac{t^{2}}{2 !}+\ldots \\
& p^{w}(t)=p_{0}^{w}+\left[\alpha_{p} M_{1}^{w}\left(t-\tau_{p}\right)-\beta_{p} p_{0}^{w}\right] t+\left[\alpha_{p} M_{2}^{w}\left(t-\tau_{p}\right)-\beta_{p} p_{1}^{w}\right] \frac{t^{2}}{2 !}+\cdots
\end{aligned}
$$

Application of the gene model without competition.

Solve the non-competitive gene regulation model to find the binding concentration of the activating protein of the non-competitive model Equation (2.6) with the initial condition (2.11)

$$
\frac{d G^{A w o}}{d t}=a_{0} G^{\prime}(t) A(t)+a r_{f} G^{A R}-a r_{0} R(t) G^{A w o}(t)-a_{f} G^{A w o}
$$

We have

$$
\begin{aligned}
& F[t]=a_{0} G^{\prime}(t) A(t)+a r_{f} G^{A R}-a r_{0} R(t) G^{A w 0}(t)-a_{f} G^{A w o} \\
& G^{\overline{A w} 0}(0)=G_{0}^{A} \\
& G_{1}^{A w o}=\left.F[t]\right|_{t=0}+g=a_{0} G_{1}^{\prime}(t) A(t)+a r_{f} G_{1}^{A R}-\left(a r_{0} R(t)+a_{f}\right) G_{0}^{A w o} \\
& G_{2}^{A w o}=\left.F^{\prime}[t]\right|_{t=0}=a_{0} G_{2}^{\prime}(t) A(t)+a r_{f} G_{2}^{A R}-\left(a r_{0} R(t)+a_{f}\right) G_{1}^{A w o}
\end{aligned}
$$

:

From the equation (3.2) we get

$$
\begin{aligned}
& G^{A w o}(t)=G_{0}^{A w o}+G_{1}^{A w o} t+G_{2}^{A w o} \frac{t^{2}}{2 !}+\ldots \\
& =G_{0}^{A w o}+\left(a_{0} G_{1}^{\prime}(t) A(t)+a r_{f} G_{1}^{A R}-\left(a r_{0} R(t)+a_{f}\right) b G_{0}^{A w o}\right) t+\left(a_{0} G_{2}^{\prime}(t) A(t)+a r_{f} G_{2}^{A R}\right. \\
& \left.-\left(a r_{0} R(t)+a_{f}\right) G_{1}^{A w o}\right) \frac{t^{2}}{2 !}+\cdots
\end{aligned}
$$

To find the repressor gene concentration take equation (2.7) with the initial condition (2.12) 


$$
\frac{d G^{R w o}}{d t}=r_{0} G^{\prime}(t) R(t)+r a_{f} G^{A R}-r a_{0} A(t) G^{R w o}(t)-r_{f} G^{R w o}
$$

We have $F[t]=r_{0} G^{\prime}(t) R(t)+r a_{f} G^{A R}-r a_{0} A(t) G^{R w o}(t)-r_{f} G^{R w o}$

$$
g=0
$$

$$
\begin{aligned}
& G^{R w o}(0)=G_{0}^{R} \quad G_{1}^{R w o}=\left.F[t]\right|_{t=0}+g=r_{0} G_{1}^{\prime}(t) R(t)+r a_{f} G_{1}^{A R}-\left(r a_{0} A(t)+r_{f}\right) G_{0}^{R w o} \\
& G_{1}^{R w o}=\left.F[t]^{\prime}\right|_{t=0}=r_{0} G_{2}^{\prime}(t) R(t)+r a_{f} G_{2}^{A R}-\left(r a_{0} A(t)+r_{f}\right) G_{1}^{R w o}
\end{aligned}
$$

:

From the equation (3.2) we get

$$
\begin{aligned}
& G^{R w o}(t)=G_{0}^{R w o}+G_{1}^{R w o} t+G_{2}^{R w o} \frac{t^{2}}{2 !}+\ldots \\
& =G_{0}^{A R}+\left(r_{0} G_{1}^{\prime}(t) R(t)+r a_{f} G_{1}^{A R}-\left(r a_{0} A(t)+r_{f}\right) G_{0}^{R w o}\right) t+\left(r_{0} G_{2}(t) R(t)+r a_{f} G_{2}^{A R}-\right. \\
& \left.\left.\left(r a_{0} A(t)+r_{f}\right) G_{1}^{R w o}\right)\right) \frac{t^{2}}{2 !}+\cdots
\end{aligned}
$$

Now, we will take the equation for the change in the concentration mRNA with competition (2.8) with the initial condition (2.13)

$$
\frac{d M^{w o}}{d t}=\alpha_{m a r} G_{\tau m}^{A R}(t)+\alpha_{m a} G_{\tau m}^{A w o}+\alpha_{m 0} G_{\tau m}^{\prime}(t)-\beta_{m} M^{w o}
$$

We have

$$
\begin{aligned}
& F[t]=\alpha_{m a r} G_{\tau m}^{A R}(t)+\alpha_{m a} G_{\tau m}^{A w o}+\alpha_{m 0} G_{\tau m}^{\prime}(t)-\beta_{m} M^{w o} \\
& g=0 \\
& M_{0}^{w o}=M(0) \\
& M_{1}^{w o}=\left.F[t]\right|_{t=0}+g=\alpha_{m a r} G_{1}^{A R}\left(t-\tau_{m}\right)+\alpha_{m a} G_{1}^{A w o}\left(t-\tau_{m}\right)+\alpha_{m 0} G_{1}^{\prime}\left(t-\tau_{m}\right)-\beta_{m} M_{0}^{w o} \\
& M_{2}^{w o}=\left.F^{\prime}[t]\right|_{t=0}=\alpha_{m a r} G_{2}^{A R}\left(t-\tau_{m}\right)+\alpha_{m a} G_{2}^{A w o}\left(t-\tau_{m}\right)+\alpha_{m 0} G_{2}^{\prime}\left(t-\tau_{p}\right)-\beta_{m} M_{1}^{w o}
\end{aligned}
$$

:

From the equation (3.2) we get

$$
\begin{aligned}
& M^{w o}(t)=M_{0}^{w o}+M_{1}^{w o} t+M_{2}^{w o} \frac{t^{2}}{2 !}+\ldots \\
& =M_{0}^{w o}+\left(\alpha_{m a r} G_{1}^{A R}\left(t-\tau_{m}\right)+\alpha_{m a} G_{1}^{A w o}\left(t-\tau_{m}\right)+\alpha_{m 0} G_{1}^{\prime}\left(t-\tau_{m}\right)-\beta_{m} M_{0}^{w o}\right) t+\left(\alpha_{m a r} G_{2}^{A R}\left(t-\tau_{m}\right)+\right. \\
& \left.\alpha_{m a} G_{2}^{A w o}\left(t-\tau_{m}\right)+\alpha_{m 0} G_{2}^{\prime}\left(t-\tau_{m}\right)-\beta_{m} M_{1}^{w o}\right) \frac{t^{2}}{2 !}+\cdots
\end{aligned}
$$

Now, as in the previous equation we will follow the same steps to find the protein concentration from the equation for the change in protein concentration of the non-competitive model (2.9) with the initial condition (2.14)

$$
\begin{aligned}
& \frac{d p^{w o}}{d t}=\alpha_{p} M_{\tau p}^{w 0}-\beta_{p} P^{w o} \\
& F[t]=\alpha_{p} M_{\tau m}^{w o}-\beta_{p} P^{w o} \\
& p_{0}^{w o}=P(0)=p_{0} \\
& p_{1}^{w o}=\left.F[t]\right|_{t=0}+g=\alpha_{p} M_{1}^{w o}\left(t-\tau_{p}\right)-\beta_{p} p_{0}^{w o} \\
& p_{2}^{w o}=\left.F^{\prime}[t]\right|_{t=0}=\alpha_{p} M_{2}^{w o}\left(t-\tau_{p}\right)-\beta_{p} p_{1}^{w o}
\end{aligned}
$$


From the equation (3.2) we get

$$
\begin{aligned}
& P^{w o}(t)=p_{0}^{w o}+p_{1}^{w o} t+p_{2}^{w o} \frac{t^{2}}{2 !}+\ldots \\
& =p_{0}^{w o}+\left(\alpha_{p} M_{1}^{w o}\left(t-\tau_{p}\right)-B_{p} p_{0}^{w o}\right) t+\left(\alpha_{p} M_{2}^{w o}\left(t-\tau_{p}\right)-\beta_{p} p_{1}^{w o}\right) \frac{t^{2}}{2 !} \\
& \quad G^{A}, G^{R} \\
& \text { mRNA }
\end{aligned}
$$

$$
C_{A^{-}}
$$

K
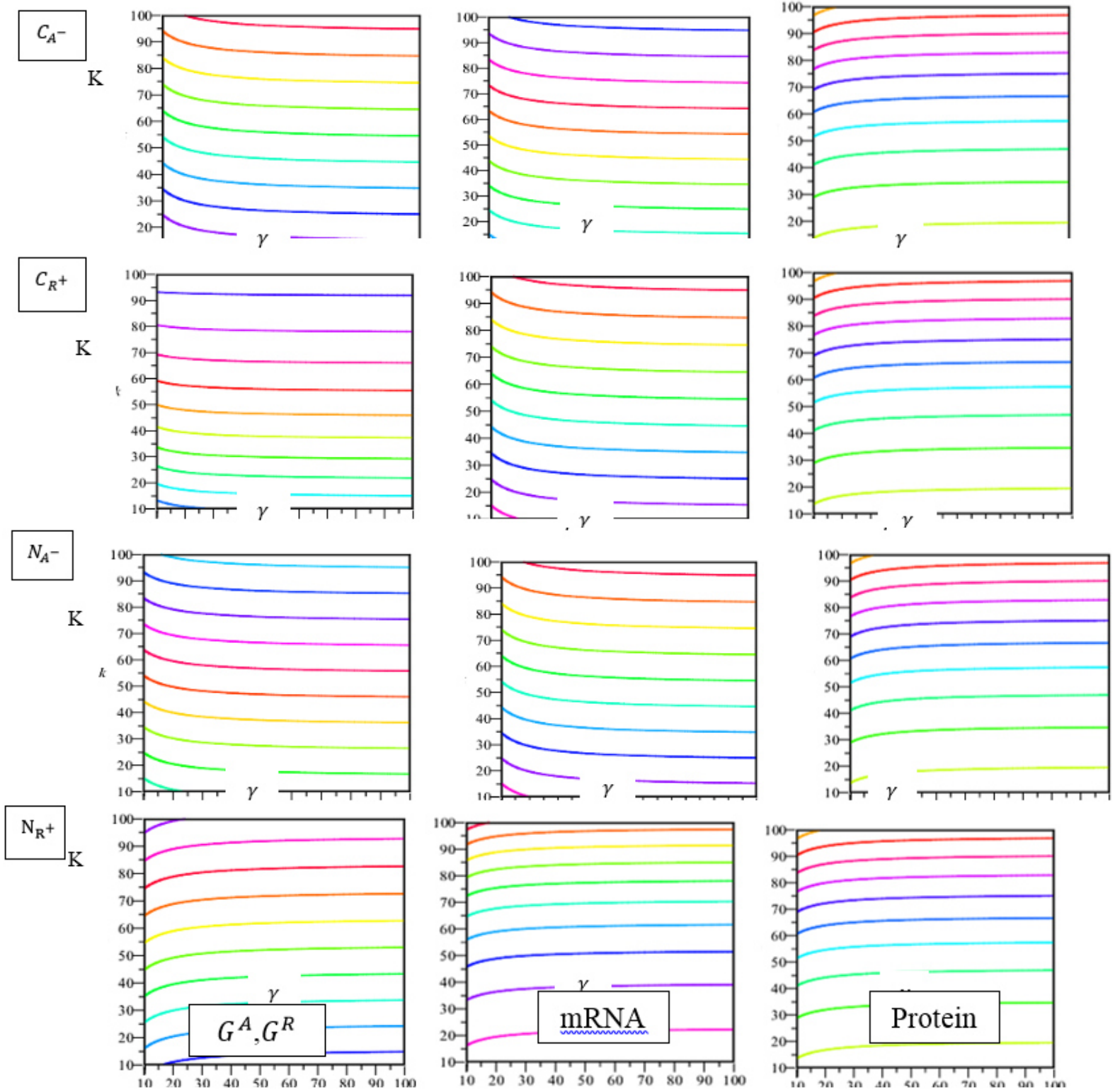

Fig1.Competitive mechanisms $\left(C_{A^{-}}, C_{R^{+}}\right)$, and noncompetitive $\left(N_{A^{-}}, N_{R^{+}}\right)$mechanisms of inhibition, $\gamma$ and k change from 10 -fold to 100 -fold when $r_{0}=10^{-4}$ 


$$
G^{A}, G^{R}
$$

\section{$C_{A^{-}}$}

k

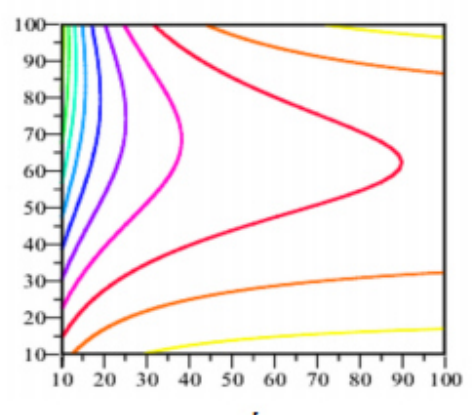

$t$

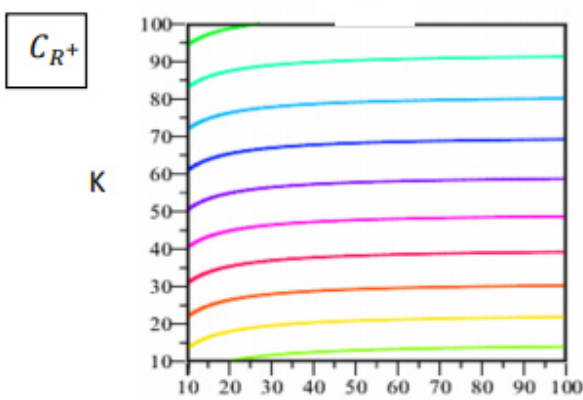

$\gamma$

$$
N_{A^{-}}
$$

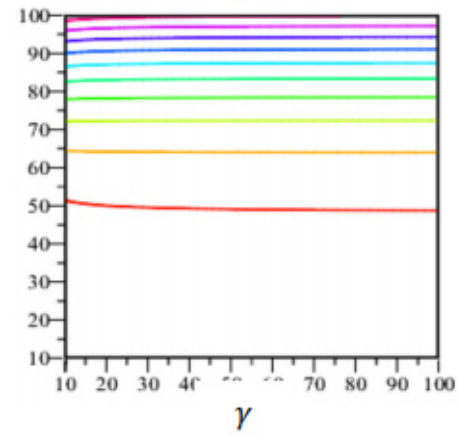

$N_{R^{+}}$

K

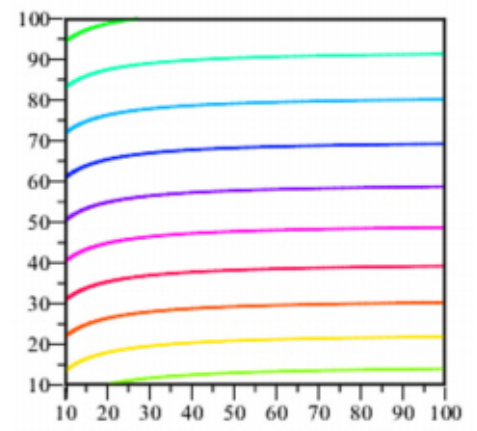

$\gamma$
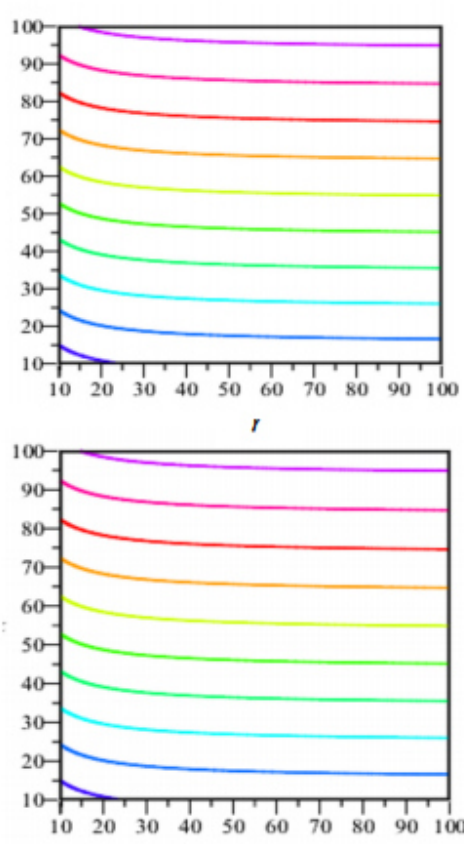

$\gamma$
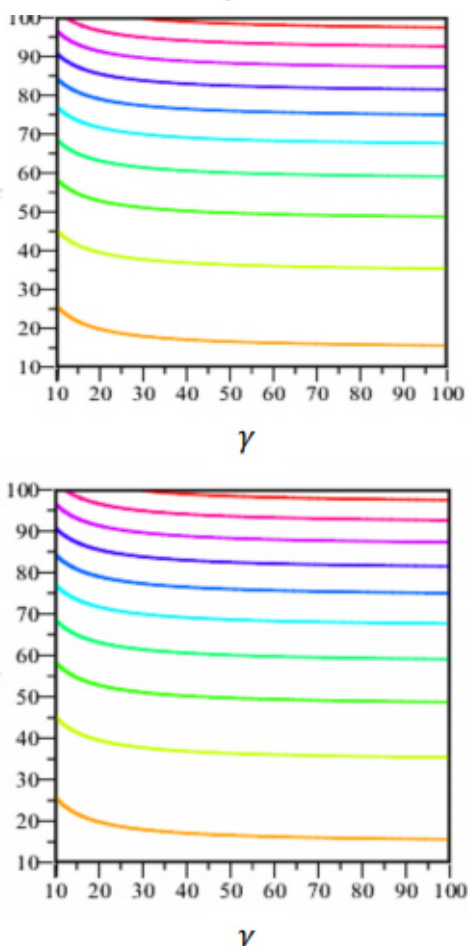

\section{Protein}
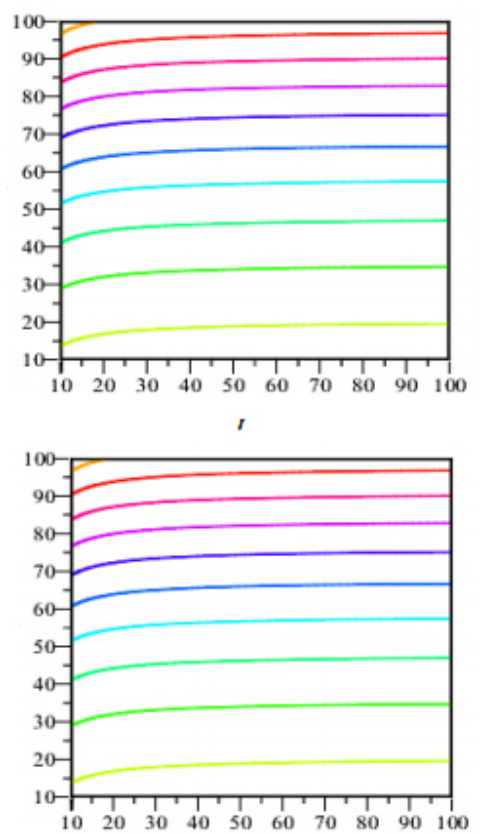

$\gamma$
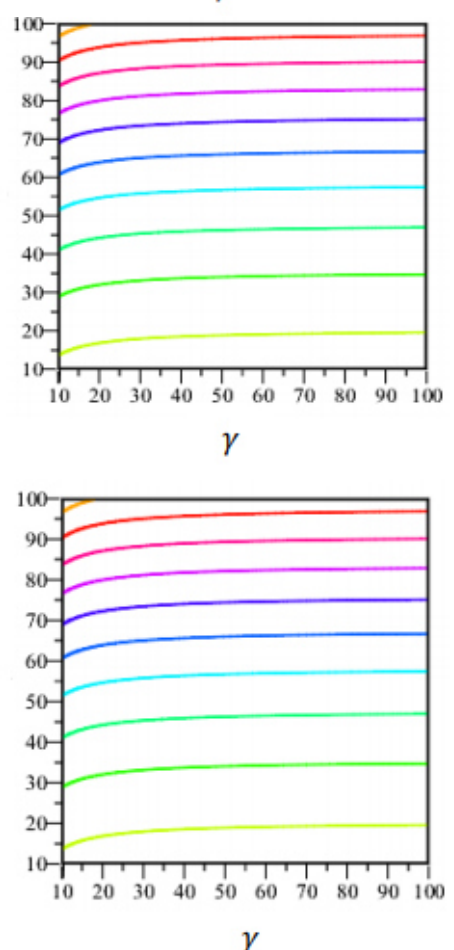

Fig2.Competitive mechanisms $\left(C_{A^{-}}, C_{R^{+}}\right)$, and noncompetitive $\left(N_{A^{-}}, N_{R^{+}}\right)$mechanisms of inhibition, $\gamma$ and k change from 10 -fold to 100 -fold when $r_{0}=10^{-3}$ 

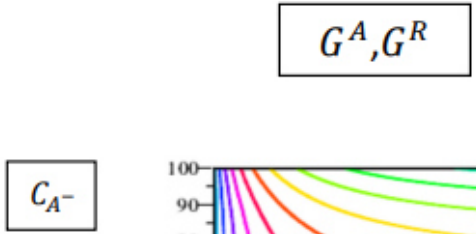

K

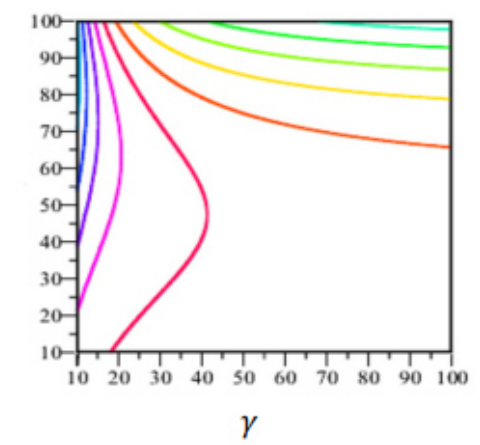

$C_{R^{+}}$

$\mathrm{K}$

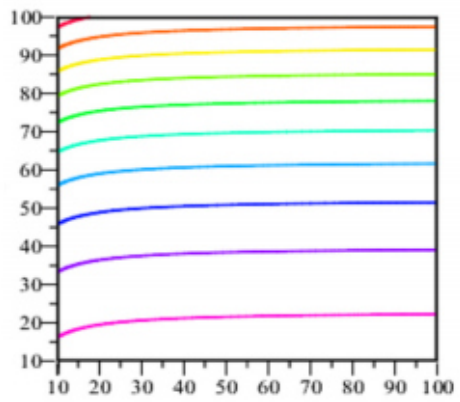

$\gamma$

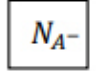

$\mathrm{K}$

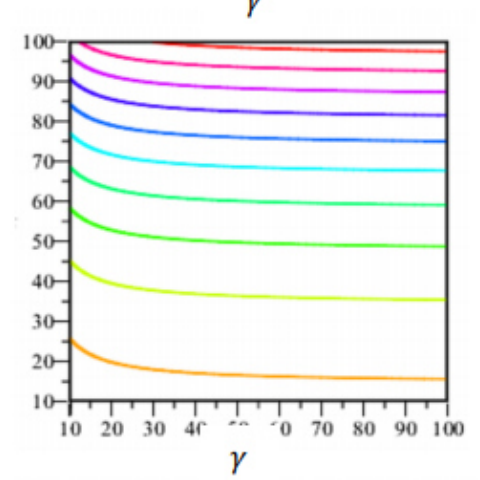

$N_{R^{+}}$

$\mathrm{K}$

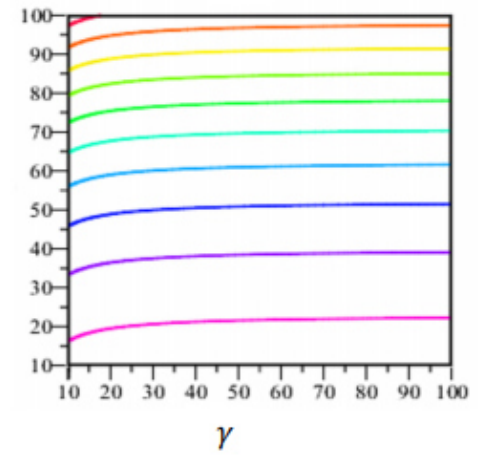

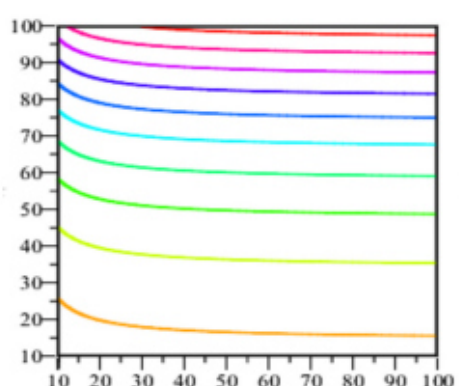

$\gamma$

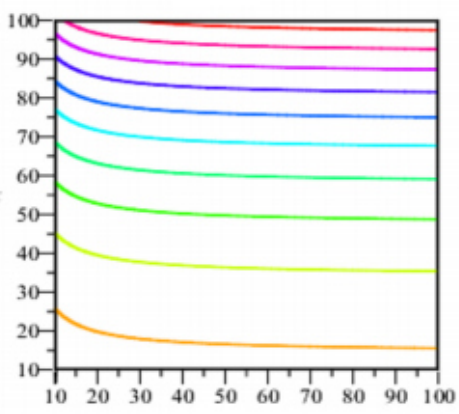

$\gamma$
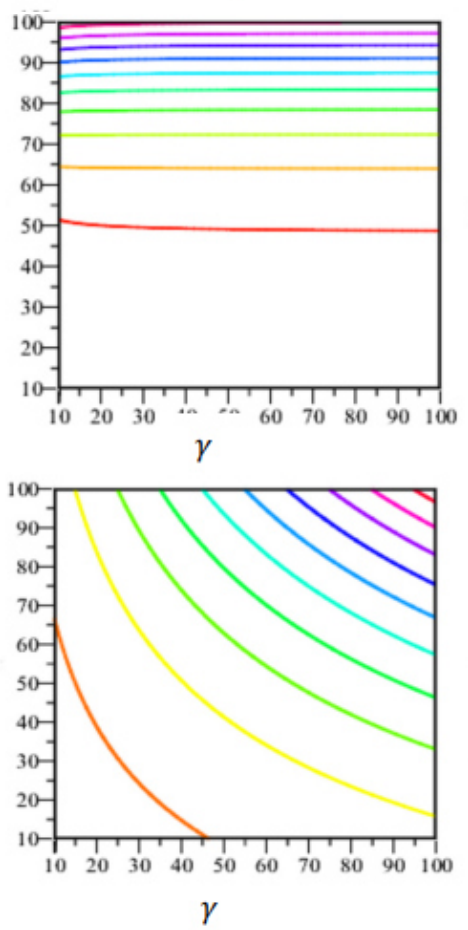

\section{Protein}
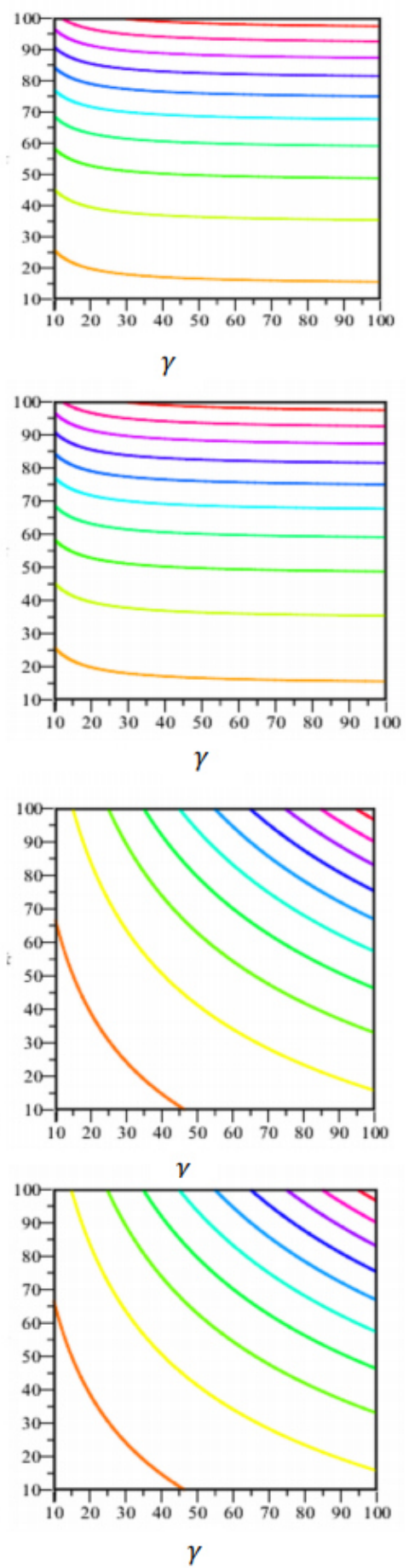

Fig3.Competitive mechanisms $\left(C_{A^{-}}, C_{R^{+}}\right)$, and noncompetitive $\left(N_{A^{-}}, N_{R^{+}}\right)$mechanisms of inhibition, $\gamma$ and k change from 10 -fold to 100 -fold when $r_{0}=10^{-2}$ 

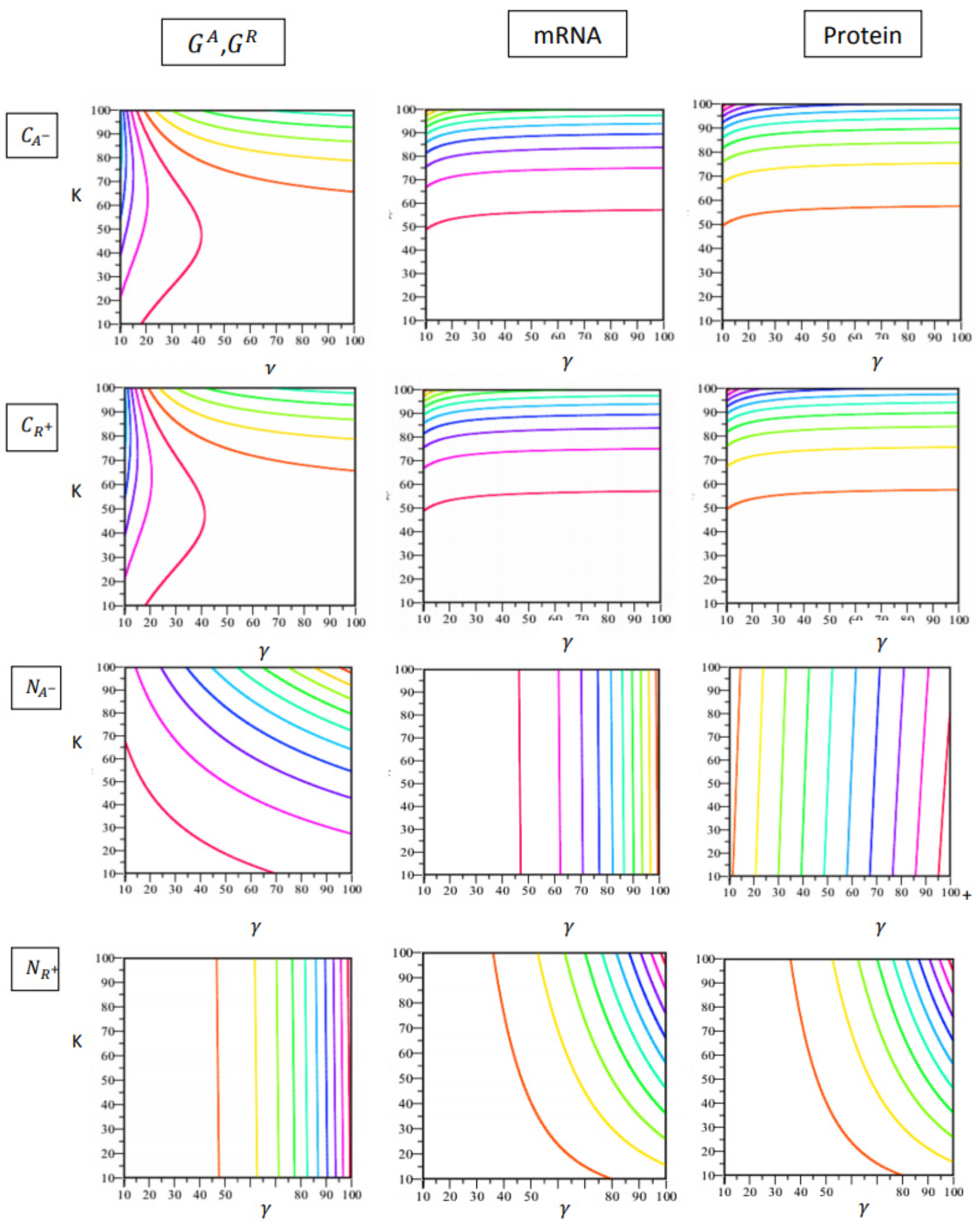

Fig4.Competitive mechanisms $\left(C_{A^{-}}, C_{R^{+}}\right)$, and noncompetitive $\left(N_{A^{-}}, N_{R^{+}}\right)$mechanisms of inhibition, $\gamma$ and k change from 10 -fold to 100 -fold when $r_{0}=10^{-1}$ 


$$
G^{A}, G^{R}
$$

$C_{A^{+}}$

K

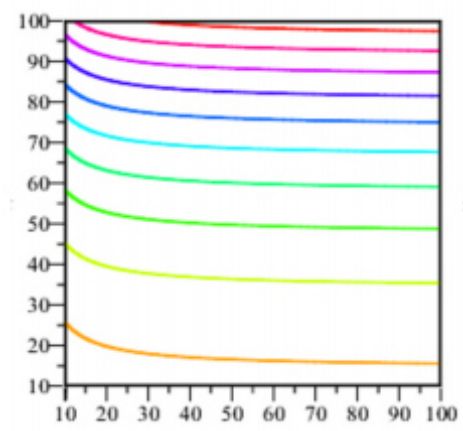

$\gamma$

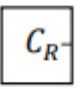

K

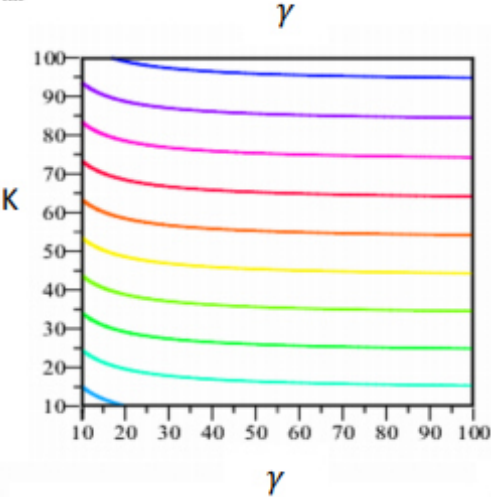

$N_{A^{+}}$

K

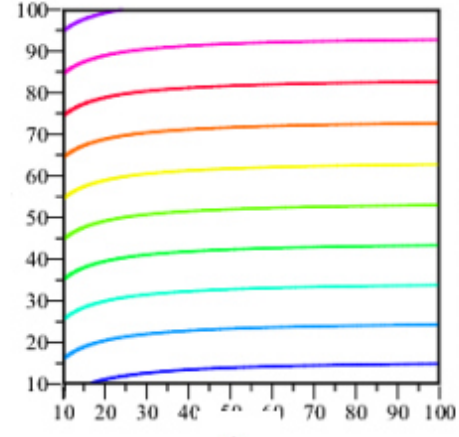

$\gamma$

$N_{R^{-}}$

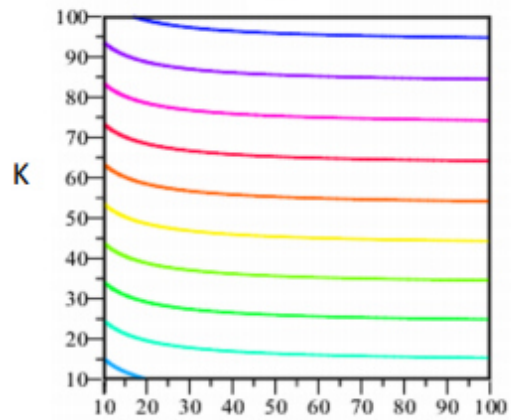

$\gamma$
mRNA

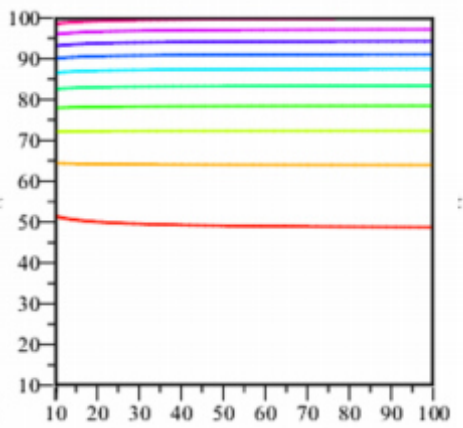

$\gamma$
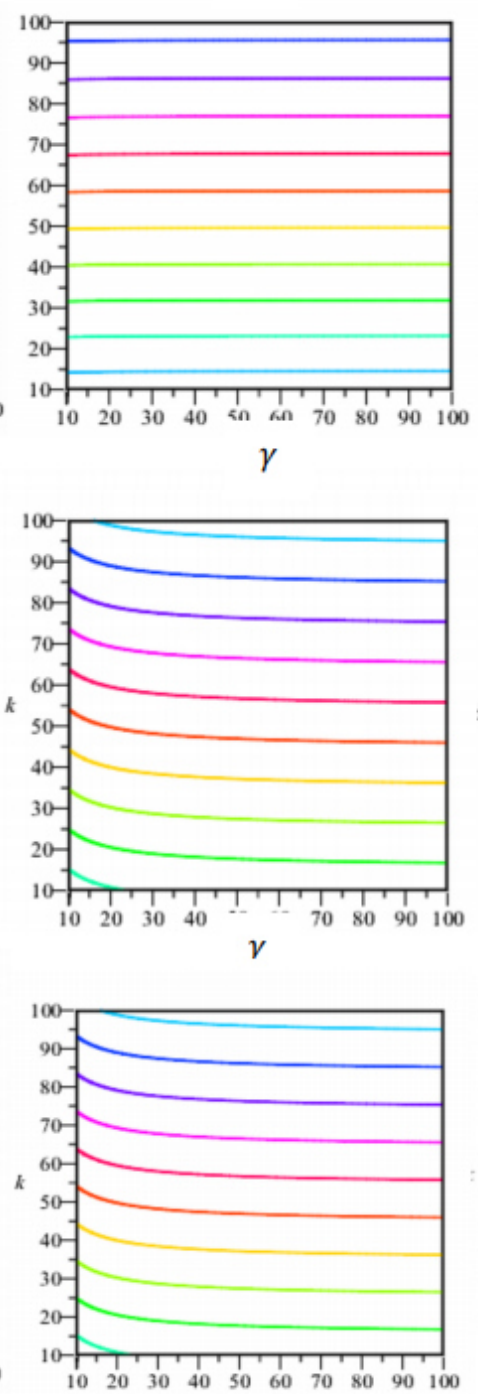

$\gamma$
Protein

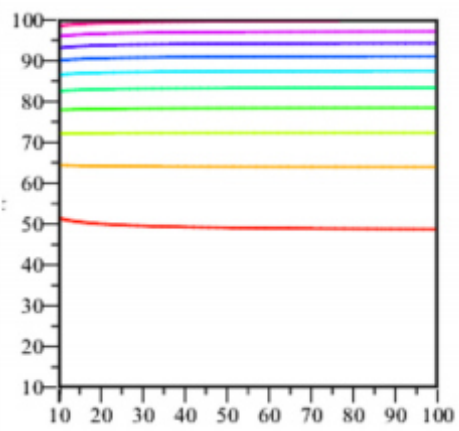

$\gamma$
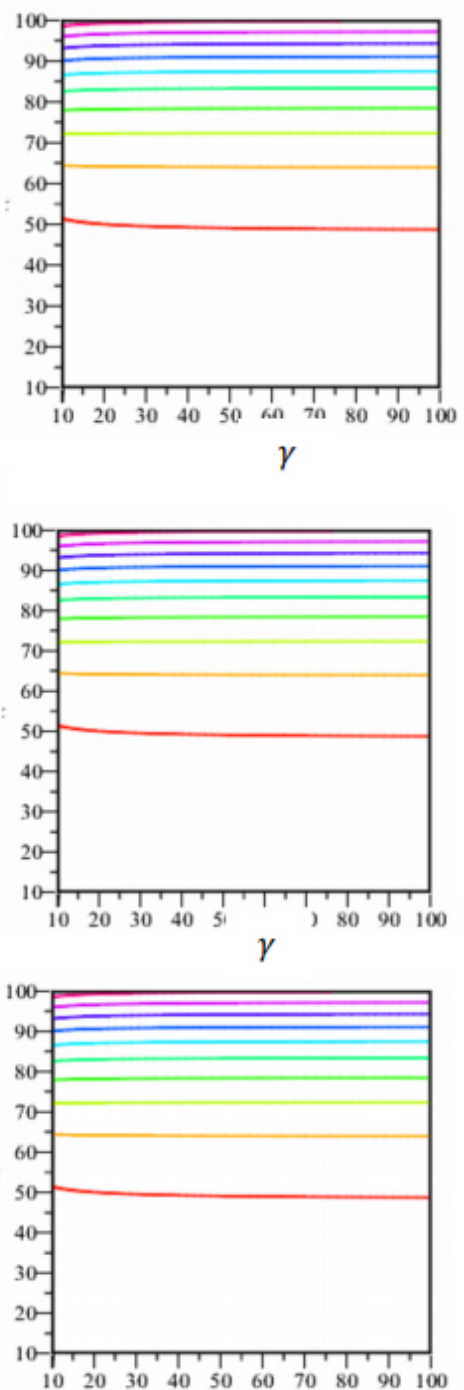

$\gamma$

Fig5.Competitive mechanisms $\left(C_{A^{+}}, C_{R^{-}}\right)$, and noncompetitive $\left(N_{A^{+}}, N_{R^{-}}\right)$Activation mechanisms,$\gamma$ and k change from 10 -fold to 100 -fold when $r_{0}=10^{-4}$ 

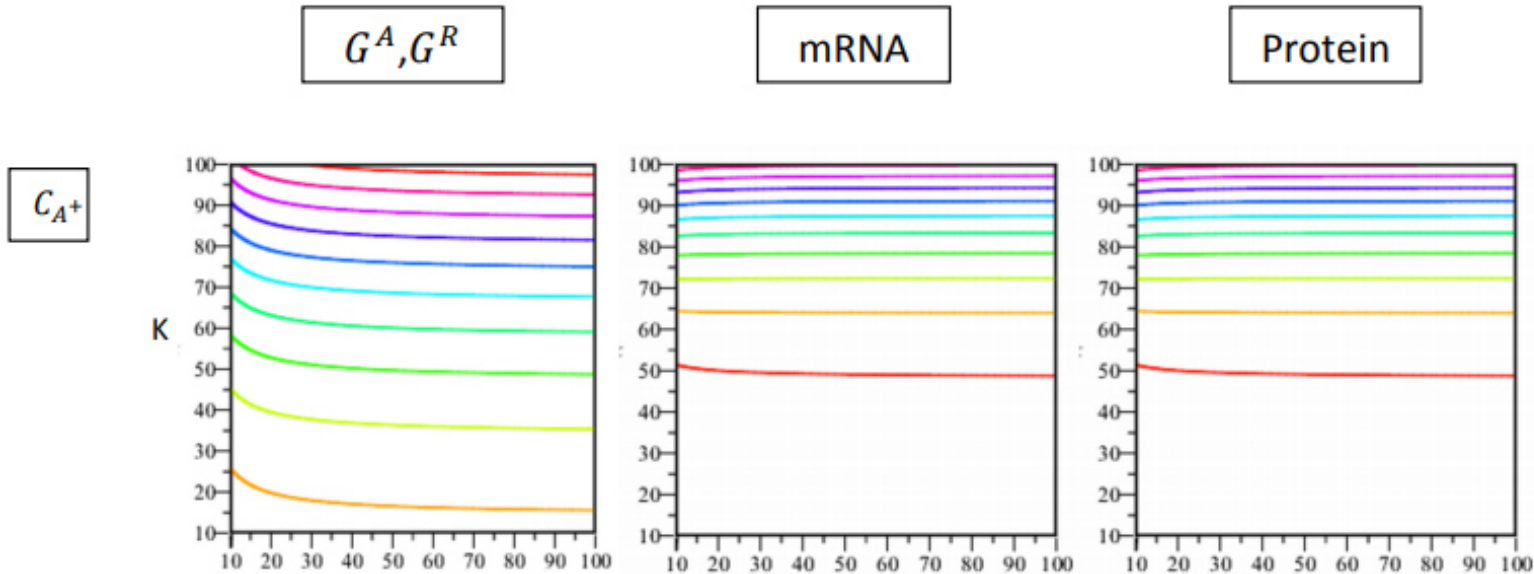

$\gamma$
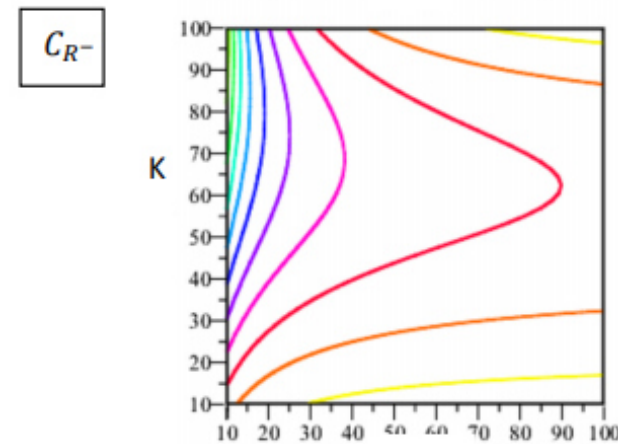

$\gamma$
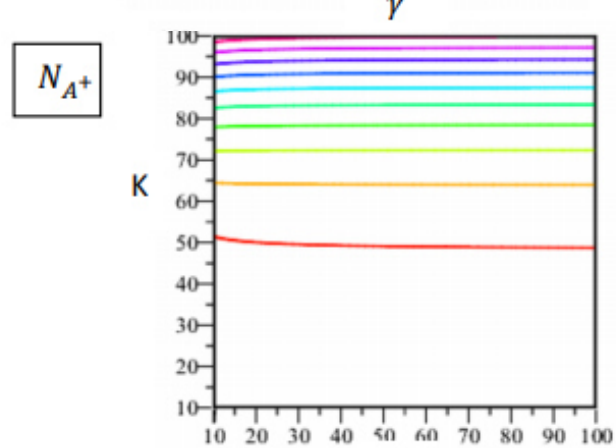

$\gamma$

$N_{R^{-}}$

K

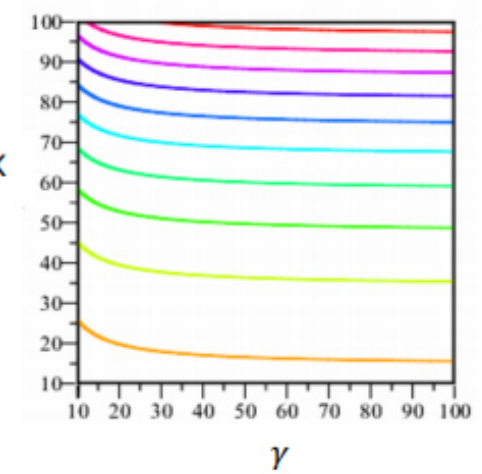

mRNA

$\gamma$

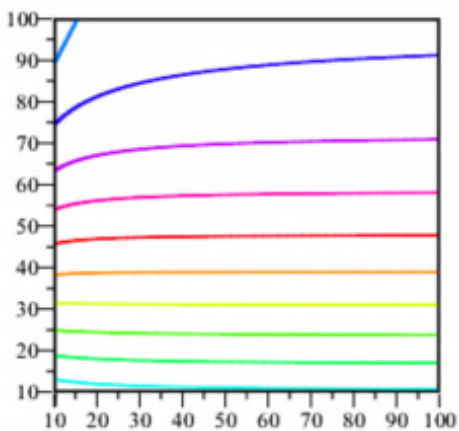

$\gamma$

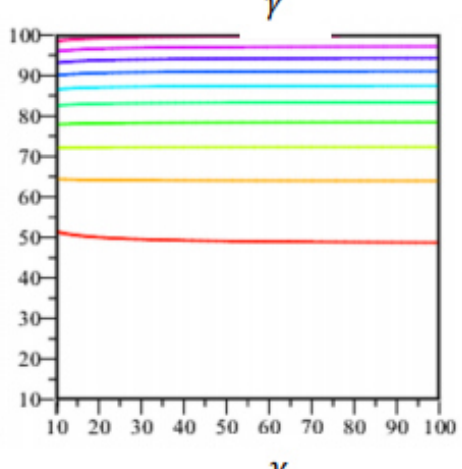

$\gamma$

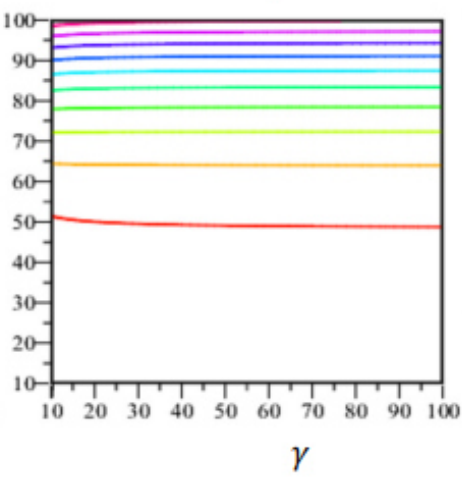

\section{Protein}
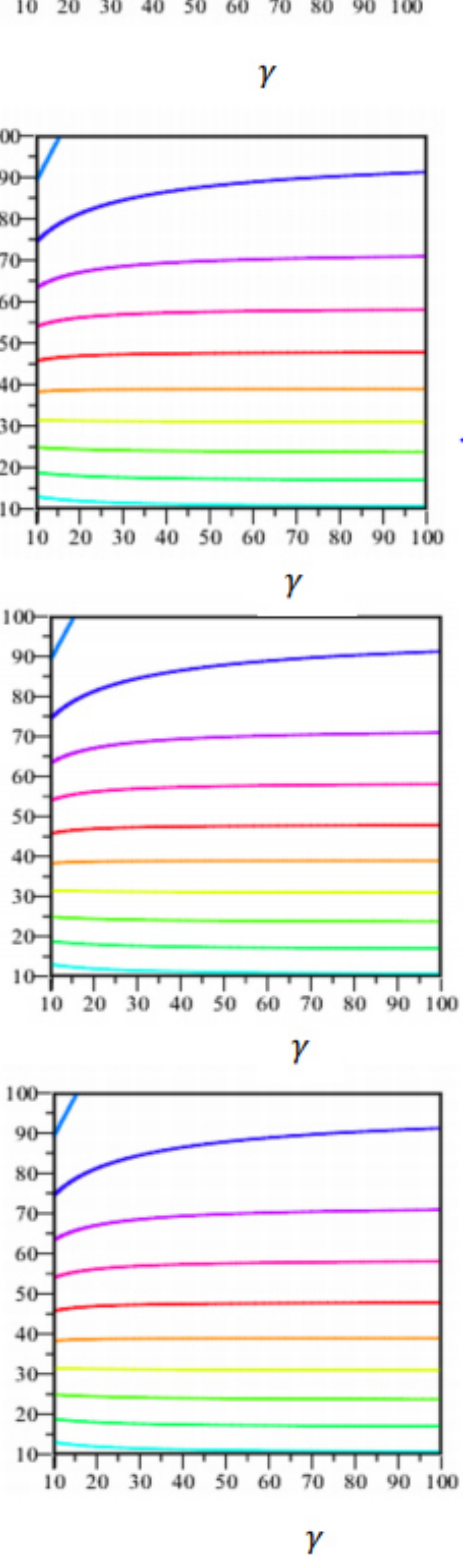

Fig6.Competitive mechanisms $\left(C_{A^{+}}, C_{R^{-}}\right)$, and noncompetitive $\left(N_{A^{+}}, N_{R^{-}}\right)$Activation mechanisms, $\gamma$ and k change from 10 -fold to 100 -fold when $r_{0}=10^{-3}$ 

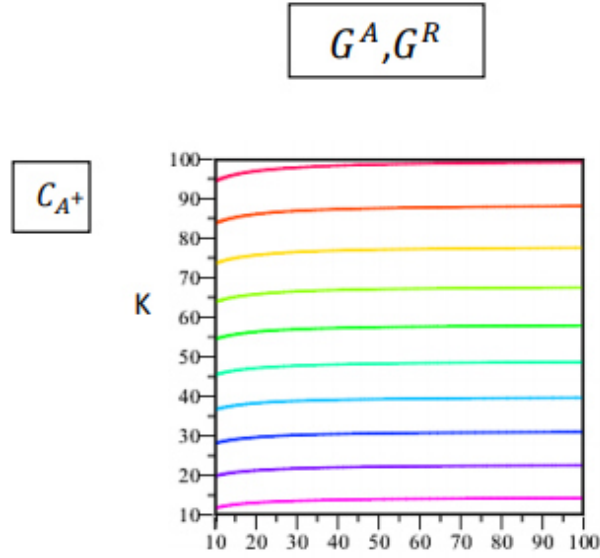

$\gamma$

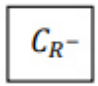

K

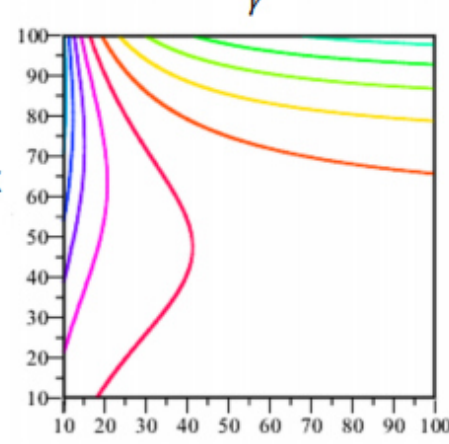

$\gamma$

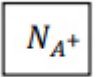

K

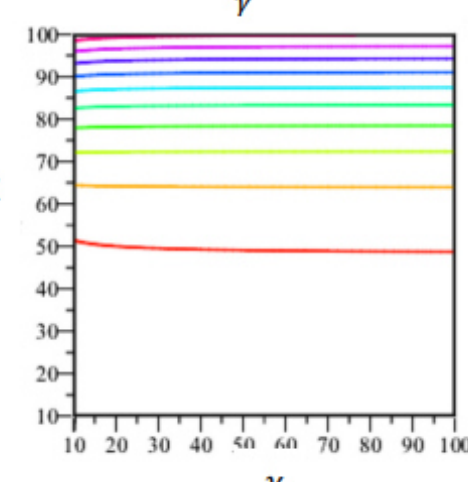

$\gamma$

$N_{R^{-}}$

K

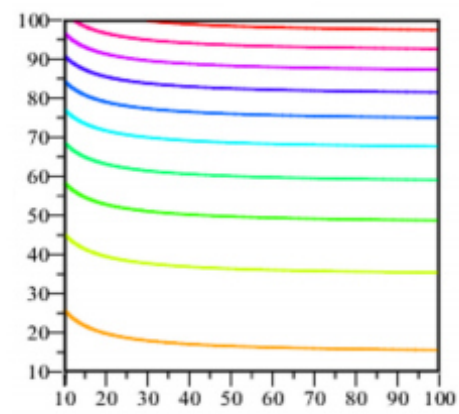

$\gamma$
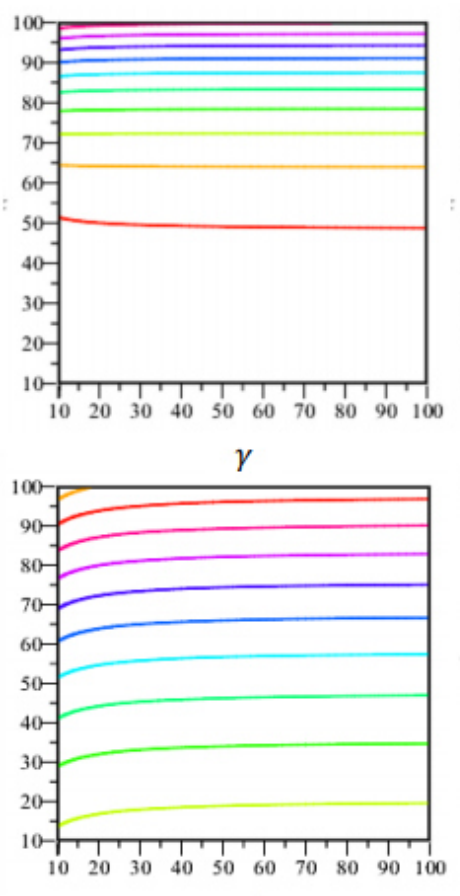

$\gamma$

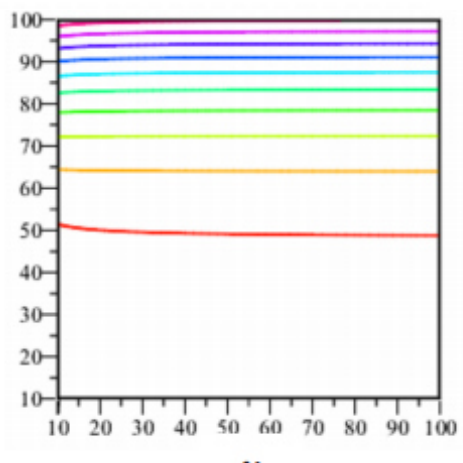

$\gamma$

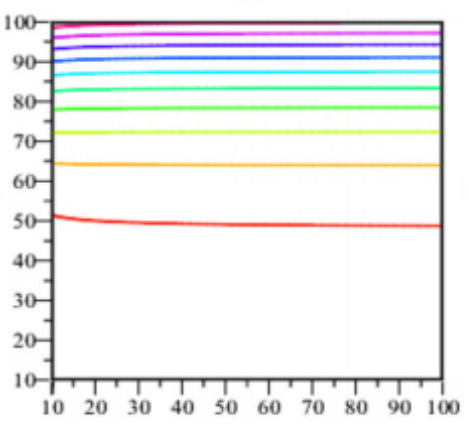

$\gamma$

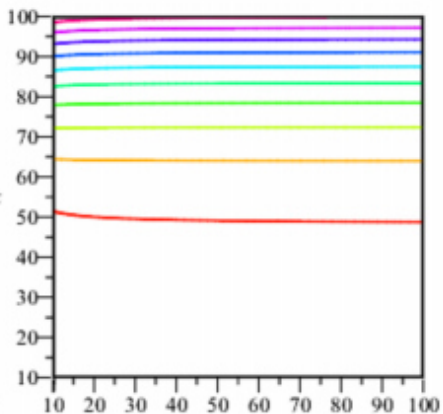

$\gamma$

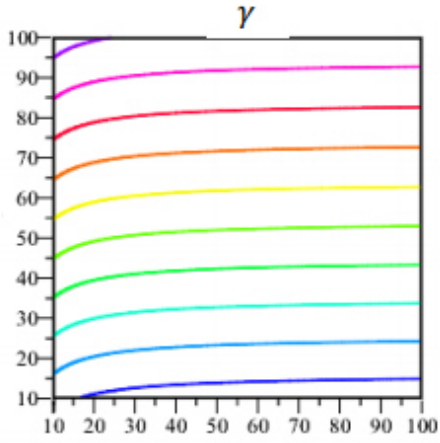

$\gamma$
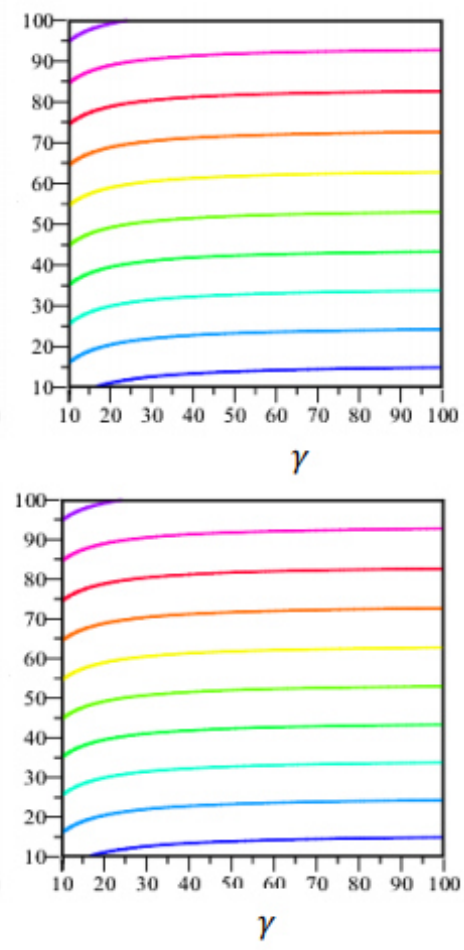

Fig7.Competitive mechanisms $\left(C_{A^{+}}, C_{R^{-}}\right)$, and noncompetitive $\left(N_{A^{+}}, N_{R^{-}}\right)$Activation mechanisms , $\gamma$ and k change from 10 -fold to 100 -fold when $r_{0}=10^{-2}$ 

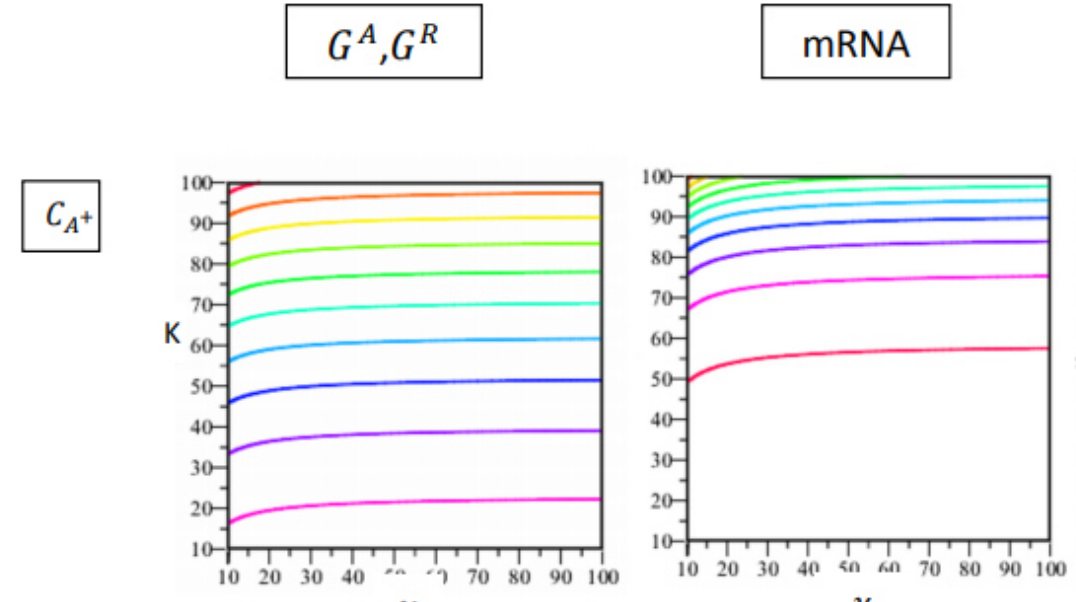

$\gamma$
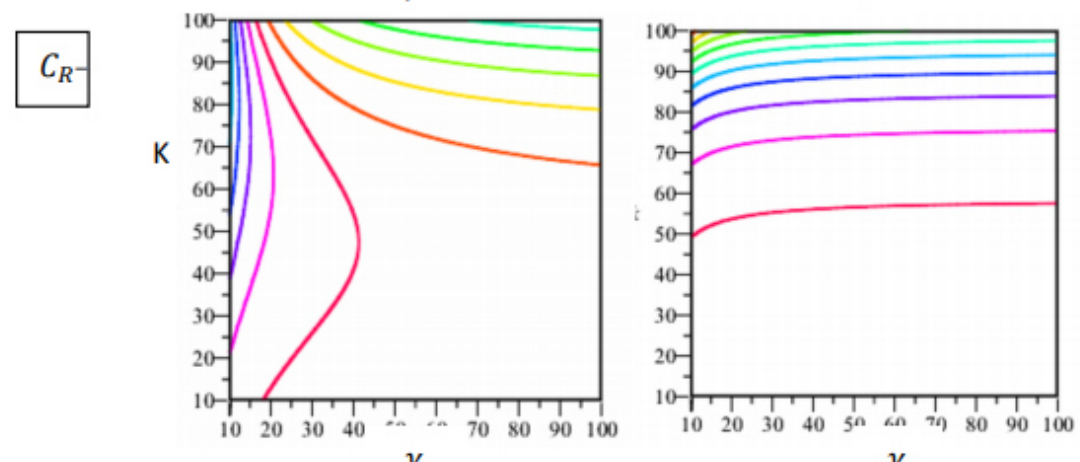

$\gamma$
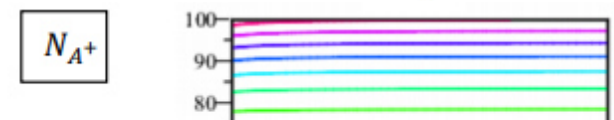

$\mathrm{K}$

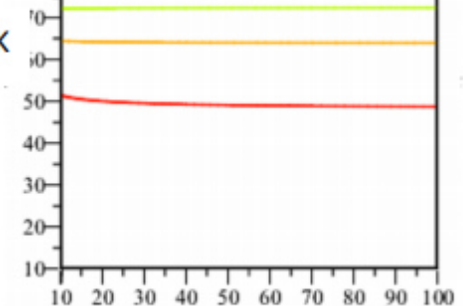

$\begin{array}{llllllllll}10 & 20 & 30 & 40 & 50 & 60 & 70 & 80 & 90 & 100\end{array}$

$\gamma$

$N_{R^{-}}$

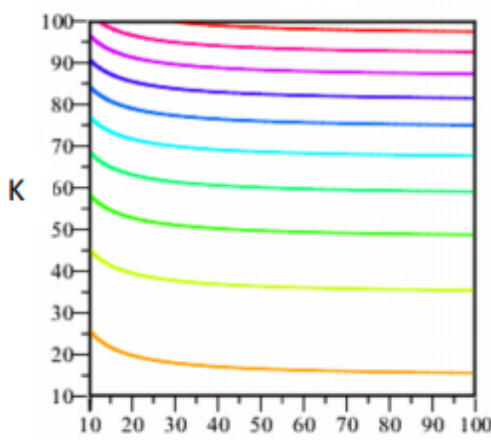

$\gamma$
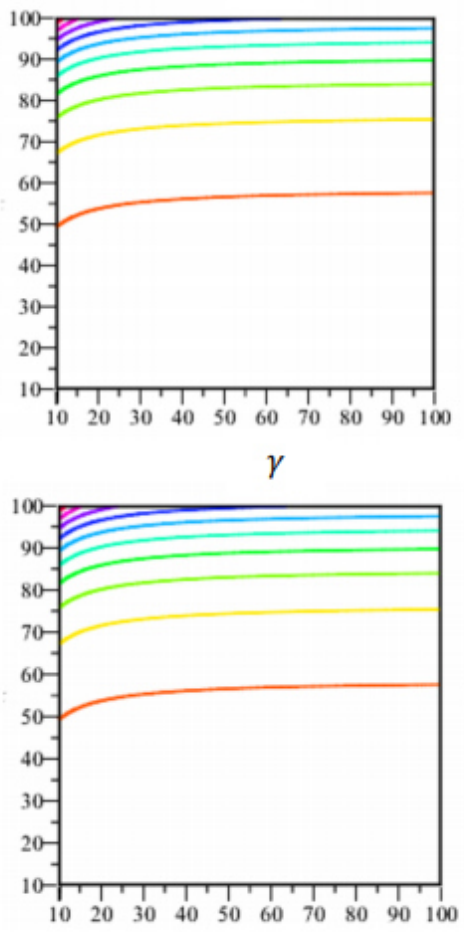

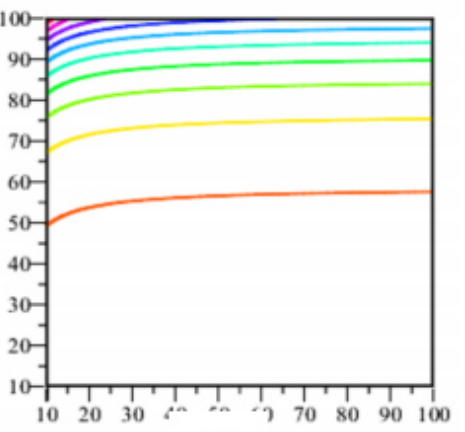

$\gamma$

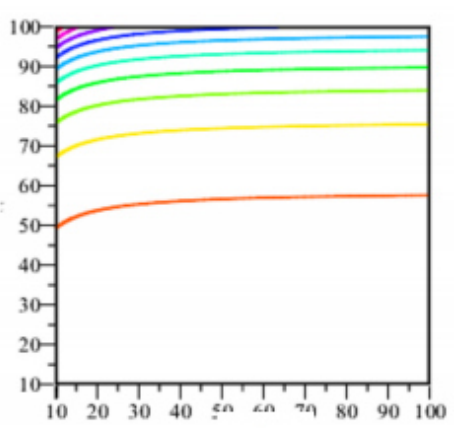

$\gamma$

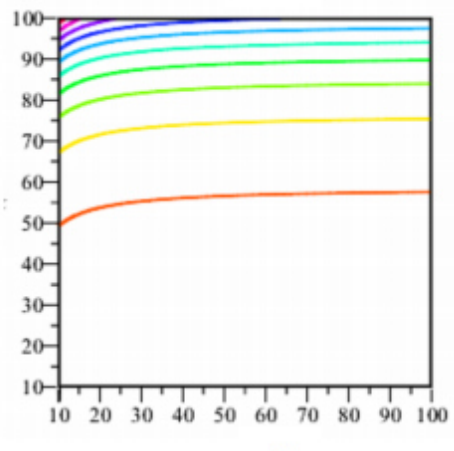

$\gamma$

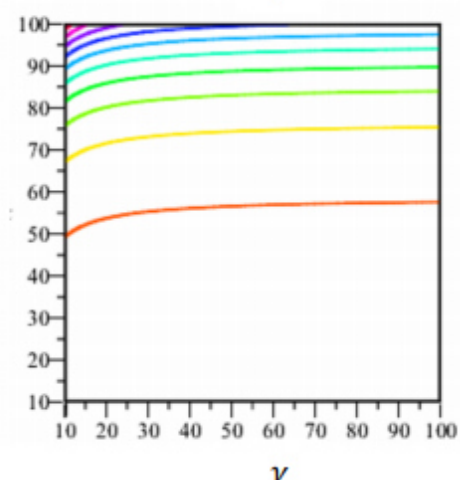

Fig8.Competitive mechanisms $\left(C_{A^{+}}, C_{R^{-}}\right)$, and noncompetitive $\left(N_{A^{+}}, N_{R^{-}}\right)$Activation mechanisms, $\gamma$ and k change from 10 -fold to 100 -fold when $r_{0}=10^{-1}$ 


\section{4-Discuss the results}

Fig 1 represents the surface graph of gene expression model solutions when $r_{0}=10^{-4}$

$C_{A^{-}}$represents the decrease of activator protein by the signal in the competitive mechanisms

$C_{R^{+}}$represents the increase of inhibitory protein by the signal in the competitive mechanisms.

$\mathrm{N}_{\mathrm{A}^{-}}$represents the decrease of activator protein by the signal in the noncompetitive mechanisms .

$N_{R^{+}}$represents the increase of inhibitory protein by the signal in the noncompetitive mechanisms .

In general, we note that the solutions are stable for $C_{A^{-}}, C_{R^{+}}, \mathrm{N}_{\mathrm{A}^{-}}$and $N_{R^{+}}$where it is almost constant with slight non-linear behavior as we can in Figure 11 where we notice that the deflection in the shape. The protein remains constant in all cases (not change with increase and decrease of the protein for competitive and noncompetitive mechanisms) where it is quasi-constant at $\gamma>20$.

Fig 2 Surface graph of gene expression model solutions when $r_{0}=10^{-3}$, there is a clear behavior of the activated gene but we note that the mRNA and protein are almost stable. The repressor gene has a lower behavior than the activator gene. In the case of non-competitive mechanisms, the activated gene $\mathrm{N}_{\mathrm{A}^{-}}$is constant at all values of $\gamma$ and $\mathrm{k}$, in addition mRNA and protein whose behavior is slightly nonlinear at $\gamma<20$. As for the repressor gene $N_{R^{+}}$ we note that at $\gamma<20$ and $\mathrm{k}>20$, suggesting that mRNA and protein behave similarly to the case mentioned above when $r_{0}=10^{-4}$.

The Surface graph of the gene expression model solution when $r_{0}=10^{-2}$ is represented in Fig 3 , in the case of competitive mechanisms, the activated gene $C_{A^{-}}$oscillates dramatically as the mRNA and protein are linear at $\gamma>40$ and $\mathrm{k}>50$, and the repressor gene $C_{R^{+}}$appears to be stable at $\gamma>30, \mathrm{k}>25$ in the case of $C_{A^{-}}$. The noncompetitive mechanisms of $\mathrm{N}_{\mathrm{A}^{-}}$(activating gene) and mRNA are linear at all values of $\gamma, \mathrm{k}$, the protein is oscillatory with all values of $\gamma$ and $\mathrm{k}$, the repressor gene $N_{R^{+}}$is semi-linear and oscillatory in the protein and mRNA.

Fig4 represents the surface graph of the gene expression model solutions when $r_{0}=10^{-2}$. In the case of $C_{A^{-}}$ competition mechanisms, the activated gene is unstable (highly fluctuating) while the mRNA and protein are linear at $\gamma>20$ and $k>70$, the repressor gene is better than the activated gene and also the mRNA and protein show the same behavior. $C_{A^{-}}$in the case of the behavior of non-competitive mechanisms $\mathrm{N}_{\mathrm{A}^{-}}$(activating gene) is fluctuating in all $\gamma, \mathrm{k}$ values while mRNA is linear and protein semi-linear, the repressor gene is opposite to the activated gene in $\mathrm{N}_{\mathrm{A}^{-}}$Linear at the repressor gene and fluctuate in mRNA and protein.

Fig5 represents the surface graph of gene expression model solutions when $r_{0}=10^{-4}$

$C_{A^{+}}$represents the increase of activated protein by signal in competitive mechanisms

$C_{R^{-}}$represents the decrease of inhibitory protein by signal in competitive mechanisms.

$N_{A^{+}}$represents the increase of activated protein by signal in non-competitive mechanisms.

$N_{R^{-}}$represents the decrease inhibited protein by signal in non-competitive mechanisms.

In general, as we notice from the graphs that the solutions are quasi-linear in all cases for all values of $k, \gamma$ from 10 to 100 where it is almost stable when $\mathrm{y}>20$.

Fig 6 represents the surface graph of gene expression model solutions when $r_{0}=10^{-3}, C_{A^{+}}$(increase activated protein), we note that the activated gene and mRNA are stable at $\gamma>30$ but in protein and mRNA the oscillatory is clear at $k<50 . C_{R^{-}}$(decreasing repressive protein) the repressor gene is significantly oscillated in all cases and the mRNA is similar to that of $C_{A^{+}}$. In the case of non-competitive mechanisms $\left(N_{A^{+}}, N_{R^{-}}\right)$the activated gene and mRNA are constant at all values $(\gamma, k)$. The repressor gene is semi-linear and is stable at $k<$ 50 for all values of $\gamma$, while the mRNA is stable for all values of $(\gamma, k)$.

In fig 7, we note that all the solutions are semi-linear or linear except for the inhibitory gene $C_{R^{-}}$(the decrease of the inhibitory protein in the competitive mechanisms) the solution is oscillatory for all values.

When looking at Fig8, we notice from the surface graph of the solutions of the gene expression model when $r_{0}=$ $10^{-1}$ that, as in Fig7, the repressor gene acts in competitive mechanisms, the mRNA and the protein are stable at $\mathrm{k}<60$ and for all values of $\gamma$. We also note that the protein in each state is stable and not affected by the change (increase or decrease of the activating and inhibitory proteins for both competitive and non-competitive states).

\section{5- Parameters}

- $\quad \beta_{\mathrm{m}}$ The mRNA degradation parameter 0.1386

- $\eta_{m a}$ and $\eta_{m 0}$ The synthesis rate constants $\eta_{m a}=1.5120$ and $\eta_{m 0}=0.1512$ 
- $\alpha_{m 0}, \alpha_{m a}$ and $\alpha_{m_{a r}}$ The synthesis rate constants of $G, G^{A}$ and $G^{A R}$

$\alpha_{m 0}=0.1584, \alpha_{m a}=1.5840$ and $\alpha_{m_{a r}}=0.4752$.

- $\quad \alpha_{P}$ protein synthesis rate constant $\alpha_{\mathrm{p}}=0.5$

- $\beta_{P}$ protein degradation rate constant $\beta_{\mathrm{p}}=0.02$.

- $\tau_{m}$ Transcriptional delay parameter $\tau_{m}=0.42$

- $\tau_{p}$ Translation delay parameter $\tau_{p}=0.46$

\section{6-Convergence analysis:}

Consider the equations (2.1-2.4) in the following form

$u\left(G^{R}, G^{A}, M, P, t\right)=G\left(u\left(G^{R}, G^{A}, M, P, t\right)\right.$

where $G$ is the linear or nonlinear operator. The solution by the present approach is equivalent to the following sequence[8]

$$
s_{n}=\sum_{i=0}^{n} u_{i}=\sum_{i=0}^{n} a_{i} \frac{(\Delta t)^{i}}{(i) !}
$$

\section{Theorem 6.1[8]:}

Let $G$ be an operator from a Hilbert space ( $H$ ) into $H$ and $u$ be the exact solution of equation (3.1). The approximate solution $\sum_{i=0}^{\infty} u_{i}=\sum_{i=0}^{\infty} a_{i} \frac{(\Delta t)^{i}}{i !}$ is Convergence to exact solution $\mathrm{u}$ when

$\exists 0 \leq \alpha<1,\left\|u_{i+1}\right\| \leq \alpha\left\|u_{i}\right\| \forall i \in \mathbb{N} \cup\{0\}$.

To analyzing and proving the convergence of approximate analytical solutions that are resulting from applying Taylor's technique to solve the gene expression model, we use the following definition and corollary that related to the above theorem[8].

\section{Definition 6.2:}

For every $n \in N \cup\{0\}$, we define

$a_{\mathrm{n}}=\left\{\begin{array}{rr}\frac{\left\|u_{n+1}\right\|}{\left\|u_{n}\right\|}\left\|u_{n}\right\| \neq 0 \\ 0 & 0, \mathrm{w}\end{array}\right.$

\section{Corollary 6.3[8]:}

From theorem (6.1) $\sum_{i=0}^{\infty} u_{i}=\sum_{i=0}^{\infty} a_{i} \frac{(\Delta t)^{i}}{i !}$ convergence to exact solution $u$ when $0 \leq \alpha_{i}<1, i=0,1,2, \ldots$ In case Gene regulation without competition To study the convergent of the activated gene $\left(G^{A}\right)$ equation (3.4), we have

$$
\begin{aligned}
& G^{A}(t)=G_{0}^{A}+\left[a_{0} A(t)\left(r f-\left(r 0 R(t)+a_{0} A(t)+r f\right) G_{0}^{\prime}-a_{f} G_{0}^{A}\right] t+\left[a _ { 0 } A ( t ) \left(-r f\left(r_{0} R(t)+a_{0} A(t)+r_{f}\right) G_{0}^{\prime}+\right.\right.\right. \\
& \left.a_{0} A(t) r_{f}-a_{f}\left(a_{0} A(t) G_{0}^{\prime}-a_{f} G_{0}^{A}\right)\right] \frac{t^{2}}{2 !}+\cdots \\
& \alpha_{1}=\frac{\left\|G^{A}{ }_{1}\right\|}{\left\|G^{A}{ }_{0}\right\|}=0.0010637289 \quad<1 \\
& \alpha_{2}=\frac{\left\|G^{A}{ }_{2}\right\|}{\left\|G^{A}{ }_{1}\right\|}=0.0019270206 \quad<1 \\
& \alpha_{3}=\frac{\left\|G^{A}{ }_{3}\right\|}{\left\|G^{A}{ }_{2}\right\|}=0.0060158144 \quad<1
\end{aligned}
$$

hence, the convergence of approximate solutions are valid .

While the convergent of the repressor gene $\left(G^{A}\right)$ equation (3.5), we have 
$G^{R}(t)=G_{0}^{R}+\left(r_{0} R(t) G_{1}^{\prime}-r_{f} G_{0}^{R}\right) t+\left(r_{0} R(t) G_{2}^{\prime}-r_{f} G_{1}^{R}\right) \frac{t^{2}}{2 !}+\ldots$ Where $G_{0}^{R}=25, \mathrm{t}=0.1$ $\mathrm{k}=10: 20$

$$
\begin{aligned}
& \alpha_{1}=\frac{\left\|G^{R_{1}}\right\|}{\left\|G^{R}{ }_{0}\right\|}=0.0085049005 \quad<1 \\
& \alpha_{2}=\frac{\left\|G^{R_{2}}\right\|}{\left\|G^{R_{1}}\right\|}=0.0077552734 \quad<1 \\
& \alpha_{3}=\frac{\left\|G^{R_{3}}\right\|}{\left\|G^{R_{2}}\right\|}=0.0041211666 \quad<1
\end{aligned}
$$

hence, the convergence of approximate solutions are valid.

To illustrate the convergent of the mRNA equation (3.6) we have

$$
\begin{aligned}
M(t)= & M_{0}+\left(\alpha_{m a r} G_{1}^{A R}\left(t-\tau_{m}\right)+\alpha_{m a} G_{1}^{A}\left(t-\tau_{m}\right)+\alpha_{m 0} G_{1}^{\prime}\left(t-\tau_{m}\right)-\beta_{m} M_{0}\right) t+\left(\alpha_{m a r} G_{2}^{A R}\left(t-\tau_{m}\right)+\right. \\
& \left.\alpha_{m a} G_{2}^{A}\left(t-\tau_{m}\right)+\alpha_{m 0} G_{2}^{\prime}\left(t-\tau_{m}\right)-\beta_{m} M_{1}\right) \frac{t^{2}}{2 !}+\cdots
\end{aligned}
$$

Where $m_{0}=25, \mathrm{t}=0.1 \quad, \gamma=20, \mathrm{k}=10: 20$

$\alpha_{1}=\frac{\left\|M_{1}\right\|}{\left\|M_{0}\right\|}=1.5260082641 \quad>1$

$\alpha_{2}=\frac{\left\|M_{2}\right\|}{\left\|M_{1}\right\|}=1.2770781353 \quad>1$

$\alpha_{3}=\frac{\left\|M_{3}\right\|}{\left\|M_{2}\right\|}=0.0007558578<1$

hence, the convergence of approximate solutions are valid at 3 iteration.

Regarding protein convergent, equation (3.7)), we have

$$
p(t)=p_{0}+\left[\alpha_{p} m_{1}\left(t-\tau_{p}\right)-\beta_{p} p_{0}\right] t+\left[\alpha_{p} m_{2}\left(t-\tau_{p}\right)-\beta_{p} p_{1}\right] \frac{t^{2}}{2 !}+\cdots
$$

Where $p_{0}=25, \mathrm{t}=0.1, \gamma=20, \mathrm{k}=10: 20$

$$
\begin{array}{ll}
\alpha_{1}=\frac{\left\|p_{1}\right\|}{\left\|p_{0}\right\|}=19.6919914481 & >1 \\
\alpha_{2}=\frac{\left\|p_{2}\right\|}{\left\|p_{1}\right\|}=5.0887080784 & >1 \\
\alpha_{3}=\frac{\left\|p_{3}\right\|}{\left\|p_{2}\right\|}=0.3333333333 & <1
\end{array}
$$

hence, the convergence of approximate solutions are valid at 3 iteration.

In case Gene regulation without competition

$$
\begin{aligned}
& G^{A}(t)=G_{0}^{A}+\left(a_{0} G_{1}^{\prime}(t) A(t)+a r_{f} G_{1}^{A R}-\left(a r_{0} R(t)+a_{f}\right) b G_{0}^{A}\right) t+\left(a_{0} G_{2}^{\prime}(t) A(t)+a r_{f} G_{2}^{A R}-\cdots\right. \\
& \left.\quad-\left(a r_{0} R(t)+a_{f}\right) G_{1}{ }^{A}\right) \frac{t^{2}}{2 !}+\cdots
\end{aligned}
$$

Where $G_{0}^{A}=25, \mathrm{t}=0.1 \quad, \gamma=20, \mathrm{k}=10: 20$

$$
\alpha_{1}=\frac{\left\|G^{{ }^{A}}{ }_{1}\right\|}{\left\|G^{A}{ }_{0}\right\|}=0.015506005<1
$$


$\alpha_{2}=\frac{\left\|G^{A_{2}}\right\|}{\left\|G^{A_{1}}\right\|}=0.00658096<1$

$\alpha_{3}=\frac{\left\|G^{A_{3}}\right\|}{\left\|G^{A_{2}}\right\|}=0.004299820<1$

hence, the convergence of approximate solutions are valid.

$G^{A}(t)=G_{0}^{A R}+\left(r_{0} G^{\prime}{ }_{1}(t) R(t)+r a_{f} G_{1}^{A R}-\left(r a_{0} A(t)+r_{f}\right) G_{0}^{R}\right) t+\left(r_{0} g_{2}(t) R(t)+r a_{f} G_{2}^{A R}-\right.$ $\left.\left.\left(r a_{0} A(t)+r_{f}\right) G_{1}^{R}\right)\right) \frac{t^{2}}{2 !}+\cdots$

Where $G_{0}^{R}=25$

, $\mathrm{t}=0.01$, $\gamma$ $=20$, $\mathrm{k}=10: 20$

$\alpha_{1}=\frac{\left\|G^{R_{1}}\right\|}{\left\|G^{R_{0} \|}\right\|}=0.008657448<1$

$\alpha_{2}=\frac{\left\|G^{{ }}{ }_{2}\right\|}{\left\|G^{{ }}{ }_{1}\right\|}=0.0077331999 \quad<1$

$\alpha_{3}=\frac{\left\|G^{R}{ }_{3}\right\|}{\left\|G^{R}\right\|}=0.0064968008<1$

hence, the convergence of approximate solutions are valid.

$$
\begin{aligned}
M(t)= & M_{0}+\left(\alpha_{m a r} G_{1}^{A R}\left(t-\tau_{m}\right)+\alpha_{m a} G_{1}^{A}\left(t-\tau_{m}\right)+\alpha_{m 0} G_{1}^{\prime}\left(t-\tau_{m}\right)-\beta_{m} M_{0}\right) t+\left(\alpha_{m a r} G_{2}^{A R}\left(t-\tau_{m}\right)+\right. \\
& \left.\alpha_{m a} G_{2}^{A}\left(t-\tau_{m}\right)+\alpha_{m 0} G_{2}^{\prime}\left(t-\tau_{m}\right)-\beta_{m} M_{1}\right) \frac{t^{2}}{2 !}+\cdots
\end{aligned}
$$

Where $m_{0}=25, \mathrm{t}=0.1, \gamma=20, \mathrm{k}=10: 20$

$$
\begin{array}{ll}
\alpha_{1}=\frac{\left\|M_{2}\right\|}{\left\|M_{1}\right\|}=1.2300895874 & >1 \\
\alpha_{2}=\frac{\left\|M_{2}\right\|}{\left\|M_{1}\right\|}=0.1794023184 & <1 \\
\alpha_{3}=\frac{\left\|M_{3}\right\|}{\left\|M_{2}\right\|}=0.1226382556 & <1
\end{array}
$$

hence, the convergence of approximate solutions are valid at 2 iteration.

$$
P(t)=p_{0}+\left(\alpha_{p} M_{1}\left(t-\tau_{p}\right)-B_{p} p_{0}\right) t+\left(\alpha_{p} M_{2}\left(t-\tau_{p}\right)-\beta_{p} p_{1}\right) \frac{t^{2}}{2 !}
$$

Where $p_{0}=25, \mathrm{t}=0.1 \quad, \gamma=20, \mathrm{k}=10: 20$

$$
\begin{array}{ll}
\alpha_{1}=\frac{\left\|p_{1}\right\|}{\left\|p_{0}\right\|}=0.5772531963 & <1 \\
\alpha_{2}=\frac{\left\|p_{2}\right\|}{\left\|p_{1}\right\|}=0.1555807855 & <1 \\
\alpha_{3}=\frac{\left\|p_{3}\right\|}{\left\|p_{2}\right\|}=0.0582840233 & <1
\end{array}
$$

hence, the convergence of approximate solutions are valid.

\section{Conclusions}

Analytical solutions and surface graphics were undertaken using (Maple 18), where we note that the competitive and non-competitive activation mechanisms in the case of increasing the abundance of the activated protein are more stable than the competitive and non-competitive activation mechanisms when reducing the abundance of the inhibitor. In the case of competitive inhibition mechanisms when increasing the abundance of the inhibitor or 
decreasing the abundance of the activating protein, we note the instability of the solutions when the value of $r_{0}$ changes from $10^{-4}$ to $10^{-1}$ in both cases, but in the case an increase in the inhibitory protein is better than a decrease in the abundance of an activated protein, because there is a large fluctuation in the solution. In the noncompetitive inhibition mechanisms, when the abundance of the activated protein is increased or the abundance of the inhibitor is reduced, the opposite happens, as there is a fluctuation in both cases, but in the case of a decrease in the abundance of the activated protein it is better. These cases are used in biological systems that require stable or unstable concentrations in order to develop or diagnose and find a treatment for a specific disease, as used according to the need of cells. Therefore, systems that need stability are better when using competitive or noncompetitive activation mechanisms, increasing their abundance activators and systems that need fluctuation in the value of concentrations are better when using inhibiting mechanisms. When comparing our solutions with the solutions obtained in [3], we find that at $r=10^{-4}$, the stability of our solutions is better in both cases (inhibition and activation mechanisms) and for the competitive and non-competitive model.

\section{References}

[1] R.R.Sarkar, R.Maithreye , and S.Sinha , Design of regulation and dynamics in simple biochemical pathways, Journal of Mathematical Biology, 63(2), 283-307, 2011.

[2] C. Wang, M. Yi, K. Yang, and L. Yang, Time delay induced transition Of gene switch and stochastic resonance in a genetic transcriptional regulatory models, BMC Systems Biology 6(Suppl 1), (9),2012.

[3]Y. Necnettin, A.M. Emin, S. Nur,A. Esra and A. Ahmet, Differential transcriptional regulation by alternatively designed mechanisms:Amathematical modeling approach. Silico Biology 12 (17) :95-127, 2016.

[4] A. Sharma and N. Adlakha, Fuzzy system model for gene expression, The Egyptian Journal of Medical Human Genetics, 19 :301-306, 2018.

[5]M. Sabah ,A new analytical approximate approach for non-linear initial value problems. Msc Thesis , University of Basrah College of Education for Pure Sciences, Department of Mathematics, 2018.

[6]A. S. J. Al-Saif and M. Sabah, A new technique for simulation the zakharov-Kuznetsov equation, Journal of advances in mathematics,J. Adv. In Mathematics,14(2):7912-7920, 2018.

[7]A. S. J. Al-Saif and M. Sabah. , Application of new simulation scheme for the nonlinear biological population model, Num. Com. Meth. Sci. Eng.,1(2), 89-99, 2019.

[8]A.G.M.Amin and M.H.Yahya, Analysis Adomain method for solving parabla partial differential equation in three dimension, Iraqi journal of statistical sciences,(20):477- 493,2011.(In Arabic)

[9] G.Adomian, A new approach to nonlinear partial differential equations. Journal of Mathematical Analysis and Applications, 102: 402-434, 1984.

[10] A.M.A. El-Sayed, S.Z. Rida and A.A.M. Arafa, Exact solutions of fractional-order biological population model, Commun. Theor. Phys., 52, 992-996, 2009.

[11]A. A. M. Arafa, S. Z. Rida and H. Mohamed, Homotopy analysis method for solving biological population model,Commun. Theor. Phys.,56(5):797-800, 2011.

[12]M.Usman, I.Rashid, T.Zubair, A.Waheed and S. T.Mohyud-Din, Homotopy analysis method for ZakharovKuznetsov (ZK) equation with fully nonlinear dispersion, Sci. Res. Essays, 8(23): 1065-1072, 2013.

[13]H. Kheiri and A. Jabbari, Homotopy analysis and Homotopy Pade' methods for two- dimensional coupled Burgers' equations, Iranian Journal of Mathematical Sciences and Informatics, 6(1): 23-31, 2011.

[14] P. Roul, Application of homotopy perturbation method to biological population model, Appl. Appl. Math., 10, 1369-1378, 2010.

[15] F. Shakeri and M. Dehghan, Numerical solution of a biological population model using He's variational iteration method, Comput. Math. Appl. 54:1197-1209, 2007.

[16] L. W. Zhang, Y. J. Deng, and K. M. Liew, An improved element-free Galerkin method for numerical modelling of biological population problems, Engineering Analysis with Boundary Elements., 40, 181-188, 2014.

[17]M.H. Heydari, Z. Avazzadeh, C. Cattani, Taylor's series expansion Method for nonlinear variable-order fractional 2D optimal control problems, Alexandria Engineering Journal ,59(6): 4737-4743,2020.

[18]S.P. Torres, J.T. Lapasió and M.G.Coque, Performance of Markers and the Homologous Series Method for Dead Time Estimation in Reversed-Phase Liquid Chromatography, Journal of Liquid Chromatography \& Related Technologies, 1065-1083, 2009.

[19] P. P. Balestrassi, A. P. Paiva, A. C.Zambroni de Souza, J. B. Turrioni and E.Popova, A multivariate descriptor method for change-point detection in nonlinear time series, Journal of Applied Statistics, 327$342,2010$.

[20] D.Luc, The Series method for random variate generation and Its application to the Kolmogorov-Smirnov distribution, American Jounal of Mathematical and Management Sciences, 1(4), 359-379, 2013. 